\title{
\begin{tabular}{l|l} 
Mibraries & DSpace@MIT
\end{tabular}
}

\author{
MIT Open Access Articles
}

Describing and prescribing the constitutive response of yield stress fluids using large amplitude oscillatory shear stress (LAOStress)

The MIT Faculty has made this article openly available. Please share how this access benefits you. Your story matters.

Citation: Dimitriou, Christopher J., Randy H. Ewoldt, and Gareth H. McKinley. “Describing and Prescribing the Constitutive Response of Yield Stress Fluids Using Large Amplitude Oscillatory Shear Stress (LAOStress)." Journal of Rheology 57.1 (2013): 27.

As Published: http://dx.doi.org/10.1122/1.4754023

Publisher: Society of Rheology

Persistent URL: http://hdl.handle.net/1721.1/79096

Version: Author's final manuscript: final author's manuscript post peer review, without publisher's formatting or copy editing

Terms of use: Creative Commons Attribution-Noncommercial-Share Alike 3.0 


\section{Describing and prescribing the constitutive response of yield stress fluids using large amplitude oscillatory stress (LAOStress)}

Christopher J. Dimitriou, ${ }^{1}$ Randy H. Ewoldt, ${ }^{2}$ and Gareth H. McKinley ${ }^{3, \text { a) }}$

1) Hatsopoulos Microfluids Laboratory, Massachusetts Institute of Technology, Cambridge, Massachusetts, 02139

${ }^{2)}$ Department of Mechanical Science and Engineering, University of Illinois at Urbana Champaign

${ }^{3)}$ Hatsopoulos Microfluids Laboratory, Massachusetts Institute of Technology, Cambridge, Massachusetts, 02139.

(Dated: May 2012)

Large Amplitude Oscillatory Shear (LAOS) is used as a tool to probe the nonlinear rheological response of a model elasto-viscoplastic material (a Carbopol microgel). In contrast to most recent studies, these large amplitude measurements are carried out in a stress-controlled manner. We outline a descriptive framework of characterization measures for nonlinear rheology under stress-controlled LAOS, and this is contrasted experimentally to the strain-controlled framework that is more commonly used. We show that this stress-controlled methodology allows for a physically intuitive interpretation of the yielding behavior of elasto-viscoplastic materials. The insight gained into the material behavior through these nonlinear measures is then used to develop two constitutive models that prescribe the rheological response of the Carbopol microgel. We show that these two successively more sophisticated constitutive models, which are based on the idea of strain decomposition, capture in a compact manner the important features of the nonlinear rheology of the microgel. The second constitutive model, which incorporates the concept of kinematic hardening, embodies all of the the essential behaviors exhibited by Carbopol. These include elasto-viscoplastic creep and time-dependent viscosity plateaus below a critical stress, a viscosity bifurcation at the critical stress, and Herschel-Bulkley flow behavior at large stresses.

a)Electronic mail: gareth@mit.edu 


\section{INTRODUCTION}

In recent years, there have been many developments in both the basic formulation of Large Amplitude Oscillatory Shear (LAOS), as well as in its applications to the study of complex fluids. On the theoretical side, there are still issues to be addressed concerning the most appropriate way to capture and represent material data, and for these reasons LAOS remains an active area of research. From a practical standpoint, the utility of LAOS as an experimental methodology is that it allows both linear and nonlinear behavior of an unknown material to be probed within a single test protocol. Moreover, the ability to independently modify the frequency and strain amplitude of the oscillations enables the mapping of an entire phase space (commonly termed the Pipkin space (Pipkin, 1972)) and provides a "rheological fingerprint" of the material behavior.

One important use of LAOS measurements is that they can be applied towards the rational development and validation of constitutive models that predict nonlinear material behavior. There have already been several examples in the rheology literature of studies focused on using LAOS to determine model parameters and test the predictive performance of constitutive models (Giacomin et al., 1993; Zhou et al., 2010; Calin et al., 2010; Ng et al., 2011; Giacomin et al., 2011; Gurnon and Wagner, 2012). Similarly, in the solid mechanics and plasticity community, experiments very similar to LAOS are frequently used to probe material behavior. Solid mechanicians will often use cyclic stress/strain loading curves (although these are often performed in tension and compression, and not in shear) in order to determine whether or not a material exhibits a particular type of behavior. One of the earliest examples of this is the study of the Bauschinger effect (Bauschinger, 1886), in which it was shown that under cyclic tension/compression metals exhibited a progressive decrease in their yield strength. This is one example of a behavior that can only be observed and quantified under cyclic loading conditions, there are numerous other phenomena that can be observed at large strains which drive the need for development of nonlinear constitutive models (Gurtin et al., 2010).

Recent developments in LAOS have primarily been focused on carrying out experiments in a strain-controlled fashion, i.e. a sinusoidal strain is imposed and in turn the periodic nonlinear stress output is measured. It is then possible to extract information about the material from this periodic stress waveform. This is typically accomplished through the use 
of Fourier analysis (Wilhelm et al., 1998; Wilhelm, 2002). Ewoldt et al. (2008) developed an ontological framework that extends the stress decomposition ideas of Cho et al. (2005) and allows one to physically interpret the higher harmonics in these periodic stress waveforms in terms of orthogonal basis functions. These basis functions capture and describe physical elements such as strain-hardening or shear-thinning in a complex material response. For compactness we refer to this as the LAOStrain framework. Both Ewoldt (2009) and Läuger and Stettin (2010) developed the corresponding framework for carrying out LAOS tests in a stress-controlled manner (or LAOStress for short). They extended some of the definitions of Ewoldt et al. (2008), but it has not yet been clearly elucidated under what conditions one may prefer to carry out LAOS experiments in a stress-controlled fashion. Läuger and Stettin (2010) demonstrate that, at large deformations, a given material can behave substantially differently under the two different cyclic loading histories. Thus, different aspects of the material behavior in the nonlinear regime may be probed using the two methods. Elegant arguments such as the ones presented by Plazek (1992) on the physical interpretation of compliances vs. moduli in the linear regime suggest that there are equally important considerations to be evaluated in LAOS.

In the present work, we motivate the use of the LAOStress framework for gels and soft solid materials that can undergo yield-like transitions. We accomplish this by showing that the nonlinear measures extracted from this framework can be used to rationally guide the development of a constitutive model that predicts the behavior of such materials, and help evaluate the relevant material parameters in a systematic fashion. This work will be primarily focused on studying elasto-viscoplastic materials, which are commonly distinguished from other complex fluids by a critical strain or stress beyond which the material transitions from a predominantly solid-like to a more liquid-like behavior (Yoshimura and Prud'homme, 1987; Doraiswamy et al., 1991). There have been debates as to whether or not the yield stress in these materials is a reality or not (see the summary provided by Barnes (1999)). Nonetheless, for many of these soft materials, the Herschel-Bulkley model (which incorporates a yield stress parameter) is often successful at capturing steady state flow behavior at sufficiently large stresses and shear rates (Meeker et al., 2004a; Coussot et al., 2009; Ballesta et al., 2008; Piau, 2007). There are also other generic types of behavior that are exhibited by elasto-viscoplastic materials, including wall slip (Barnes, 1995) and many studies have focused on understanding the nature of this slip and how it relates to the microstructure of 
a material (Gibaud et al., 2009; Ballesta et al., 2008; Meeker et al., 2004b). Thixotropy is another frequently encountered behavior which some soft elasto-viscoplastic materials may exhibit (Barnes, 1997; Coussot et al., 2002; Møller et al., 2006). Recently, the presence of thixotropy has been promulgated as a distinguishing behavior which separates a subclass of "ideal" yield stress fluids from other classes of yielding materials (Møller et al., 2009).

Large amplitude oscillatory shear has already been utilized as a method to study these types of fluids (Ewoldt et al., 2007, 2010; Hess and Aksel, 2011; Rogers et al., 2011b,a). Recently the work by Rogers et al. (2011b) described the response of soft yielding materials (specifically a colloidal star polymer) to LAOS as a sequence of physical processes. The authors introduced the concept of a "Cage Modulus" - a measure that captures elastic behavior during a particular part of the LAOS cycle. They show that under strain-controlled conditions this modulus cannot be evaluated in terms of the higher harmonics in the Fourier transform of the stress signal. This suggests that there may be benefits towards taking a stress-controlled approach towards LAOS, because the zero stress compliance $\left(J_{M}^{\prime}\right)$ defined empirically in the work by Ewoldt (2009) and Läuger and Stettin (2010) is inversely connected to the concept of a "Cage Modulus" discussed by Rogers et al. (2011b,a). Comparatively little work has been done in using LAOStress to evaluate these types of nonlinear measures and distinguish between the predictions of different constitutive models for elastoviscoplastic materials (such as the models of Saramito (2009) and Fielding et al. (2009) for example).

The specific complex fluid studied in the present work is a Carbopol microgel. This soft solid has been studied extensively in recent years and is a canonical examples of an "ideal" yield stress fluid that exhibits little to no thixotropy (see Piau (2007) for a good review, as well as Møller et al. (2009); Møller et al. (2009); Coussot et al. (2009)). The lack of thixotropy in these microgels has made them ideal candidates for rheological studies. In the present work, the material measures obtained with LAOStress are contrasted to those of the strain-controlled framework (LAOStrain), and we discuss the differing physical interpretations provided by these two sets of measures. Specifically, the compliance $J_{M}^{\prime}$ measured in the limit of zero imposed stress is shown to be a good indicator of the intracycle elasticity of the material, and only exhibits a weak variation over a wide range of frequencies and stress amplitudes. Guided by the stress-controlled LAOS measurements, we develop two constitutive models that predict the rheological behavior of these soft solid materials. These 
rheological equations of state capture a distinct sequence of processes under large amplitude deformation, consistent with that described by Rogers et al. (2011b): in particular, under oscillatory stress loading, the response involves an initial elastic straining, followed by viscoplastic flow, and a subsequent elastic unloading when the applied stress is decreased. The second, more complex, constitutive model discussed here is also capable of exhibiting a viscosity bifurcation at a critical stress. Below this critical stress the model exhibits an unbounded power-law-like growth in the viscosity over time, whereas at constant applied stresses above this critical value, it converges to the familiar Herschel-Bulkley steady state flow curve that is commonly seen for these types of materials. This nonlinear rheological behavior can be described in terms of a constitutive process known as kinematic hardening, which is discussed in detail. These models are both formulated using a decomposition of strain - an approach commonly taken in the continuum mechanics and plasticity literature (Gurtin et al., 2010). Thus, an added benefit of using these models is that they can easily be generalized to frame invariant 3D tensorial form using the multiplicative Kroner decomposition of the deformation gradient, F(x,t) (Kroner, 1960; Gurtin et al., 2010).

\section{EXPERIMENTAL}

\section{A. Materials}

The material used for this study is a $0.5 \%$ wt. Carbopol microgel (Carbopol 901 variant manufactured by Lubrizol). This class of microgel has been used extensively in many other studies (Roberts and Barnes, 2001; Piau, 2007) and it has been demonstrated to behave as an "ideal" yield stress fluid in the sense that it is not strongly thixotropic (Møller et al., 2009). The steady state flow behavior of Carbopol microgels is typically well predicted by the powerlaw Herschel-Bulkley flow rule (Coussot et al., 2009; Oppong and de Bruyn, 2007; Divoux et al., 2010) provided that wall-slip is eliminated, and is relatively easy to measure due to the lack of thixotropy exhibited by Carbopol. For these reasons, Carbopol microgels serve as very useful model fluids for exploring and quantifying the LAOStress framework. The microstructure of these gels typically consists of highly swollen and compressed sphericalshaped blobs (of characteristic dimension $\sim 5 \mu \mathrm{m}$ ) which, at high enough concentrations, provide the material with its yield stress (Ketz et al., 1988; Carnali and Naser, 1992; Piau, 
2007; Mahaut et al., 2008).

\section{B. Rheometry}

All strain-controlled oscillatory measurements were carried out on an ARES straincontrolled rheometer (TA Instruments), while stress-controlled oscillatory measurements were carried out using an ARG2 rheometer (TA Instruments). Cone and plate geometries were used in all cases, in order to impose uniform strain fields within the sample. Carbopol microgels are well known to experience wall slip when subjected to steady shearing deformations (Roberts and Barnes, 2001; Coussot et al., 2009; Barral et al., 2010) so for repeatable measurements of true material response it is essential to minimize wall slip. Roughened test fixtures (created using adhesive-backed sandpaper with controlled grit size) are therefore used on both rheometers (with root mean squared roughness $R_{q} \sim 30 \mu \mathrm{m}$ ). The effectiveness of this roughening was verified using two techniques: first, it was observed that the roughened surfaces led to increased values of the measured yield stress in the fluid (Barnes (1995) showed that this indicates a change in the degree of slip in the material); secondly, it was possible to obtain direct velocimetric measurements of the flow field within the microgel undergoing steady shearing using a Rheo-PIV apparatus developed previously (Dimitriou et al., 2011, 2012). These velocimetric measurements show that when the gel is in contact with the roughened surfaces, wall slip is effectively eliminated.

One important issue that arises when controlling an oscillatory deformation on a sample with a stress-controlled rheometer is the potential impact that the instrument rotational inertia may have on the measurements (Läuger and Stettin, 2010). Specifically, for most single head rheometers (such as the ARG2) the total torque signal measured by the rheometer can be additively decomposed into two components; the sample torque and the inertia torque. For progressively larger torque amplitudes and oscillation frequencies, the maximum acceleration experienced by the rotating fixture assembly grows rapidly. As a result, the inertial contributions to the torque increase and may become large enough to dominate the total torque signal. For these reasons, all stress-controlled oscillatory shear experiments were carried out at sufficiently small frequencies and stress amplitudes such that instrument inertia did not significantly affect the total torque signal. A more detailed discussion on the effects of instrument inertia is provided in section B of the appendix. 


\section{THEORY}

\section{A. Stress vs. Strain Decomposition for a Yielding Material}

We first motivate our discussion of the stress-controlled LAOS framework by providing an illustrative example of experimental data. In Fig. 1 below, we show the response of the Carbopol microgel to a sinusoidally imposed strain, $\gamma=\gamma_{0} \sin \omega t$ when the strain amplitude $\gamma_{0}$ is small (linear region) and when $\gamma_{0}$ is large (nonlinear region).
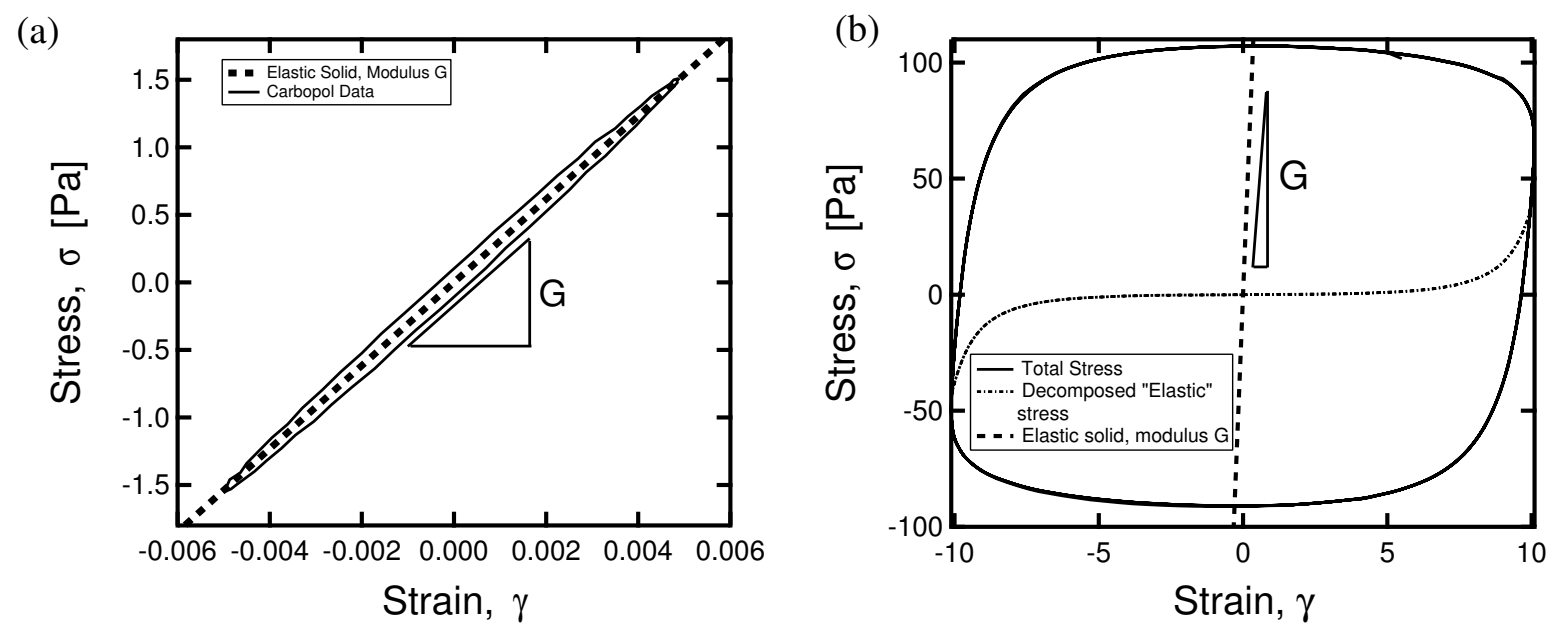

FIG. 1. Comparison of Carbopol response to small and large amplitude oscillatory deformations. Data was collected in controlled strain mode, at a frequency of $\omega=0.5 \mathrm{rad} / \mathrm{s}$.

As can be seen in Fig. 1 (a), for small amplitudes $\left(\gamma_{0}=0.5 \%\right)$ the response of the Carbopol microgel to the cyclic strain loading is primarily elastic (with elastic modulus $G=300 \mathrm{~Pa}$ indicated on the plot) and little dissipation due to the low value of $G^{\prime \prime}=12 \mathrm{~Pa}$. At the large strain amplitude $\left(\gamma_{0}=1000 \%\right)$, the response of the material is qualitatively different. The shape of the cyclic stress-strain loading curve is closer to a rectangle than an ellipse. This is representative of the response expected for an elasto-viscoplastic material as discussed previously by Ewoldt et al. (2010); Hess and Aksel (2011) and Rogers et al. (2011b). Using the strain-controlled LAOS framework, the total shear stress $\sigma(t)$ (which is a periodic signal containing multiple higher harmonic components) can be decomposed into two contributions, an "elastic" stress $\sigma^{\prime}$ which is a single-valued function of $x=\gamma / \gamma_{0}$ (the scaled strain), and a "viscous" stress $\sigma^{\prime \prime}$ which is a single-valued function of $y=\dot{\gamma} / \dot{\gamma}_{0}$ (the scaled strain rate). The decomposition defined by Cho et al. (2005) and used by Ewoldt et al. (2008) therefore is: 


$$
\begin{aligned}
\sigma^{\prime} & \equiv \frac{\sigma(\gamma, \dot{\gamma})-\sigma(-\gamma, \dot{\gamma})}{2} \\
\sigma^{\prime \prime} & \equiv \frac{\sigma(\gamma, \dot{\gamma})-\sigma(\gamma,-\dot{\gamma})}{2}
\end{aligned}
$$

The fact that the "elastic" stress $\sigma^{\prime}$ and the "viscous" stress $\sigma^{\prime \prime}$ are single-valued functions of strain and strain rate respectively allows one to fit various functional forms to the two stresses. These functional forms (such as the orthogonal Chebyshev polynomials used by Ewoldt et al. (2008)) can then be used to understand the type of intracycle nonlinearities exhibited by the material over the course of one oscillation. While this decomposition is mathematically sound, the physical interpretation of the decomposition may vary from material to material (Rogers and Lettinga, 2012). In particular, in Fig. 1 (b), we see that the decomposed "elastic" stress for Carbopol is apparently very low for strain values $-7 \leq \gamma \leq 7$ This seems to be at odds with what is known about the linear, elastic behavior of the gel at low strain amplitudes. The dashed line showing the stress-strain curve for an elastic solid with modulus $G=300 \mathrm{~Pa}$ further emphasizes this discrepancy. What is apparent is that the zero strain modulus characterizing the decomposed "elastic" stress-strain curve is much smaller (by several orders of magnitude in fact) than the actual shear modulus $G$ of the Carbopol measured in the small deformation limit. This contradictory behavior is difficult to interpret, especially if one wishes to understand the behavior of the material in terms of a sequence of physical processes, as argued by Rogers et al. (2011b) and Rogers and Lettinga (2012). It would also be confusing to use polynomial fitting coefficients of the decomposed $\sigma^{\prime}$ curve to extract information about the elastic behavior of the Carbopol for oscillatory deformations when $\gamma_{0}$ is large. Indeed, Rogers and Lettinga (2012) showed that one could interpret the LAOS response of the the Elastic Bingham model studied by Yoshimura and Prud'homme (1987) (in which a linear elastic behavior is present) as exhibiting strain stiffening - an inherently nonlinear elastic behavior. We can identify a similar apparent trend in the Carbopol microgel from the upturned shape of the decomposed elastic stress-strain curve shown in Fig. 1 (b) at large strains.

In order to better understand the behavior of the Carbopol system at these large strain amplitudes, we first consider a simple elastic-perfectly plastic constitutive model (Lemaitre and Chaboche, 1990), that approximately captures the behavior of the material in Fig. 1. A 
critical feature of this model is the additive decomposition of the shear strain $\gamma$ into elastic and plastic components:

$$
\gamma=\gamma^{e}+\gamma^{p}
$$

Where the elastic strain $\gamma^{e}$ is related through the shear stress $\sigma$ as follows:

$$
\sigma=G \gamma^{e}
$$

The plastic strain rate, $\dot{\gamma}^{p}$ is assumed to be zero for stresses below a critical yield stress $|\sigma|<\sigma_{y}$ (i.e. there is no plastic flow below the yield stress). When the stress in the material reaches the yield stress $\sigma_{y}$, the material yields, resulting in the stress saturating and plastic flow occurring in the same direction as the stress. For this simple model the plastic flow is rate independent - i.e. the stress is constant for all values of the plastic strain rate $\dot{\gamma}^{p}$. This constitutive model can be thought of as an elastic element in series with a yielding element, with elastic strain increasing linearly with the stress, and plastic strain only accumulating for values of $|\sigma|=\sigma_{y}$, the yield stress. The rate-independent plasticity behavior is somewhat poorly defined (for example, the behavior of this model is not defined for applied stresses of $|\sigma|>\sigma_{y}$ ) however rate-independent flow can be realized through the following limit of a power-law flow rule:

$$
\frac{\sigma}{\sigma_{y}}=\lim _{m \rightarrow 0}\left(\dot{\gamma}^{p}\right)^{m}
$$

The response of such a model to an oscillatory deformation $\gamma=\gamma_{0} \sin \omega t$ is shown in Fig. 2 . Beginning from the left hand point (s) on the cyclic curve at zero stress, the material first undergoes an elastic deformation. When the stress reaches the yield stress $\sigma_{y}$ it saturates, and rate-independent plastic flow begins to occur. When the direction of straining is reversed, elastic unloading occurs and the stress begins to drop below the yield stress $\sigma_{y}$.

It can be seen that this model successfully captures the idea that under an increase in the imposed strain $\gamma(t)$, the material will undergo a sequence of physical processes. These processes are specifically those of elastic loading, rate independent plastic flow, and elastic unloading. The decomposition of the strain $\gamma$ into elastic $\left(\gamma^{e}\right)$ and plastic $\left(\gamma^{p}\right)$ components means that the elastic stress-strain curve (which is just a plot of $\sigma$ vs. $\gamma^{e}$ ) can be overlaid on the cyclic loading curve (shown as a dashed line on Fig. 2).

It is also possible to use symmetry arguments similar to those made by Cho et al. (2005) 


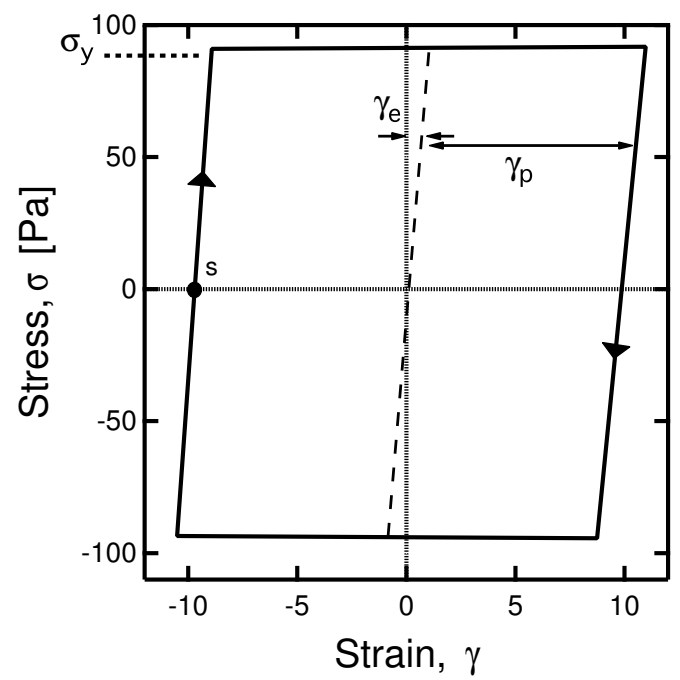

FIG. 2. Response of a simple elasto-plastic model to oscillatory shear strain $\gamma=\gamma_{0} \sin \omega t$. The yield stress corresponds to the location of the plateau in the cyclic curve with a value of $\sigma_{y}=90$ Pa.

to extract an apparent elastic strain $\gamma^{\prime}(\sigma)$ (note the functional dependency on the imposed stress $\sigma$ ) from the measured total strain field $\gamma(t)$ using the following definition:

$$
\gamma^{\prime}(\sigma)=\frac{\gamma\left(t_{1}\right)+\gamma\left(t_{2}\right)}{2} \quad \text { where } \sigma\left(t_{1}\right)=\sigma\left(t_{2}\right)
$$

Note that for this particular constitutive model, the apparent elastic strain $\gamma^{\prime}(\sigma)$ determined from the strain decomposition of Eq. 5 (which assumes nothing about the underlying nature of the constitutive model) is exactly equal to $\gamma^{e}(\sigma)$ of the constitutive model given in Eq. 2 . More generally, from the shape of the Lissajous curves shown in Figs. 1, 2, it is clear that this decomposition more faithfully captures the elastic nature of the material response at small stresses. One cannot prove that the identity $\gamma^{e}(\sigma) \equiv \gamma^{\prime}$ holds in general for all constitutive models, however these decomposed strain curves strongly suggest that, at least for yield-like elasto-viscoplastic materials, it is more desirable to decompose the measured experimental strain data into individual elastic and plastic components, rather than the corresponding stress data. The decomposed strains can then be fit to a particular functional form. For example, the decomposed elastic strain $\gamma^{e}(\sigma)=\gamma^{\prime}(\sigma)$ in Fig. 2 can be easily fit to a linear function, with slope equal to the elastic modulus $G$ from Eq. 3.

We apply this strain decomposition to the Carbopol data presented in Fig. 1, in order to obtain the apparent elastic strain $\gamma^{\prime}(\sigma)$ of the material. The resulting strain decomposition 
is shown in Fig. 3; clearly when we compare this elastic stress-strain curve to that of Fig. 1 (b), we see that the variation in the apparent elastic strain $\gamma^{\prime}(\sigma)$ in Fig. 3 is in much closer agreement with the stress-strain curve of the linear elastic solid (dashed line with modulus $G)$ at low stresses. Given what is known separately about the behavior of the Carbopol at low stresses from Fig. 1 (a) (i.e. it can be roughly approximated as an elastic solid with modulus $G$ ), the elastic stress-strain curve $\gamma^{\prime}(\sigma)$ obtained in Fig. 3 is thus a better indicator of how the material behaves elastically over the course of the loading cycle.

The disadvantage of carrying out this strain decomposition when the total shear strain $\gamma(t)$ is the input to the system $\left(\gamma=\gamma_{0} \sin \omega t\right)$ is that there is no guarantee that the timeresolved decomposed strain signal $\gamma^{\prime}$ is "linear", i.e. it will likely contain multiple higher harmonics. For this reason, we describe a framework in the next section that allows stresscontrolled experiments to be carried out, with total shear stress as the input $\left(\sigma=\sigma_{0} \cos \omega t\right)$ and with the measured strain $\gamma$ (the output) being decomposable into elastic and plastic components.

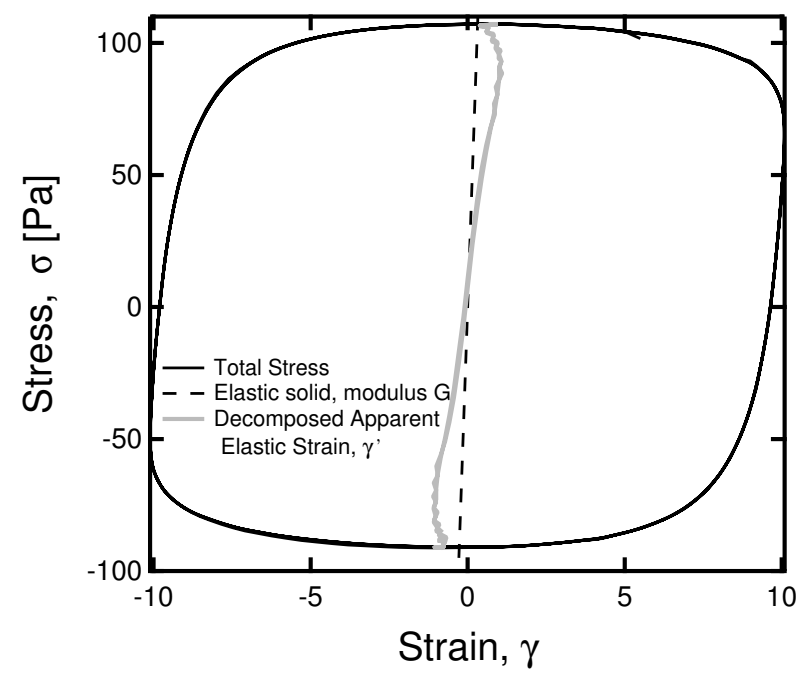

FIG. 3. Decomposed apparent elastic strain $\gamma^{\prime}$ of the Carbopol gel as defined through Eq. 5 during a strain-controlled LAOS test with $\gamma_{0}=10$. The slope of this decomposed curve is in better agreement, especially at small strains, with the shear modulus of a linear elastic solid with modulus $G$ shown by the dashed line. 


\section{B. Stress-controlled LAOS framework for analysis of experimental data}

The framework used in this paper is similar to that used by Ewoldt (2009) and Läuger and Stettin (2010) with some slight changes in convention. We also provide complementary definitions of the analogous Chebyshev coefficients to those used by Ewoldt et al. (2008) for the strain-controlled case. We begin by noting that we impose a sinusoidal stress on the material:

$$
\sigma(t)=\sigma_{0} \cos \omega t
$$

The resulting strain $\gamma\left(t ; \sigma_{0}, \omega\right)$ can then be decomposed into a Fourier series as follows:

$$
\gamma\left(t ; \sigma_{0}, \omega\right)=\sum_{n \text { odd }}\left\{J_{n}^{\prime}\left(\omega, \sigma_{0}\right) \sigma_{0} \cos n \omega t+J_{n}^{\prime \prime}\left(\omega, \sigma_{0}\right) \sigma_{0} \sin n \omega t\right\}
$$

We consider responses in which only odd harmonics are present. This was also the approach taken by Ewoldt et al. (2008). Even harmonics may arise during transient responses (Atalik and Keunings, 2004) or due to the presence of dynamic wall slip (Graham, 1995); however we will not consider these types of phenomena in this work. If desired, it is straightforward to define the coefficients for even values of $n$ using the formalism we describe. We also neglect any constant strain offsets (corresponding to the $n=0$ term in Eq. 7) that may arise in the response of the material due to initial nonlinear transients in the startup of the imposed oscillatory shear stress. These offsets depend on the specific material and the amplitude of the imposed stress, but in the present framework we will only consider the long time periodic oscillating state with the intracycle strain $\gamma(t)$ being defined relative to the average strain value evaluated over one full cycle. Furthermore, the convention of using a cosine wave for the imposed stress is adopted, because it simplifies the subsequent expressions used to determine Chebyshev fitting coefficients.

Based on the fact that there are only odd harmonics present in the strain signal represented in Eq. 7, we can then decompose our strain into an apparent elastic strain, $\gamma^{\prime}$, and an apparent plastic strain, $\gamma^{\prime \prime}$ :

$$
\begin{aligned}
\gamma^{\prime}(t) & =\sigma_{0} \sum_{n \text { odd }} J_{n}^{\prime}\left(\omega, \sigma_{0}\right) \cos n \omega t \\
\gamma^{\prime \prime}(t) & =\sigma_{0} \sum_{n \text { odd }} J_{n}^{\prime \prime}\left(\omega, \sigma_{0}\right) \sin n \omega t
\end{aligned}
$$


The strain decomposition is based on the idea that we desire an apparent elastic strain, $\gamma^{\prime}$, such that over one cycle of oscillation $\gamma^{\prime}$ is a single-valued function of $\sigma$. We also desire an apparent plastic strain, $\gamma^{\prime \prime}$, such that its time derivative, the plastic strain rate $\dot{\gamma}^{\prime \prime}$, is a single-valued function of $\sigma$. The convention of naming $\gamma^{\prime}$ an elastic strain and naming $\gamma^{\prime \prime}$ a plastic strain then follows because we usually think of elastic strain as depending on the imposed stress only, and the plastic strain rate during flow as depending on stress only. It can be shown that the decomposition represented by Eqns. 8 and 9 is unique, in the sense that no other linear decomposition of $\gamma$ satisfies the properties of $\gamma^{\prime}$ being a single-valued function of $\sigma$ and $\dot{\gamma}^{\prime \prime}$ being a single-valued function of $\sigma$ (see Appendix C). However we also emphasize that for a general class of constitutive models in which strain is decomposable into elastic and plastic components (denoted $\gamma^{e}$ and $\gamma^{p}$ respectively), the strain decomposition represented by Eqns. 8 and 9 into apparent elastic and plastic contributions $\left(\gamma^{\prime}\right.$ and $\left.\gamma^{\prime \prime}\right)$ will not necessarily yield the true elastic and plastic strains $\gamma^{e}$ and $\gamma^{p}$ defined through the constitutive model. In the previous section we saw that that the true and apparent strains coincided for a simple elasto-plastic constitutive model, however in Sec. IV D we will discuss an example of a constitutive model in which $\gamma^{e}$ and $\gamma^{p}$ differ from $\gamma^{\prime}$ and $\gamma^{\prime \prime}$. For these reasons we use the modifier "apparent" to distinguish between the experimentally-measurable strains and the constitutive counterparts.

Having defined $\gamma^{\prime}$ and $\gamma^{\prime \prime}$, we can follow the reasoning of Ewoldt et al. (2008) and represent these single-valued functions of stress as a series of orthogonal Chebyshev polynomials $T_{n}(x)$, where $x$ is the scaled stress, $x=\sigma(t) / \sigma_{0}$.

$$
\begin{aligned}
& \gamma^{\prime}(t)=\sigma_{0} \sum_{n \text { odd }} J_{n}^{\prime}\left(\omega, \sigma_{0}\right) \cos n \omega t=\sigma_{0} \sum_{n \text { odd }} \underbrace{J_{n}^{\prime}\left(\omega, \sigma_{0}\right)}_{c_{n}} T_{n}(x) \\
& \dot{\gamma}^{\prime \prime}(t)=\sigma_{0} \sum_{n \text { odd }} n \omega J_{n}\left(\omega, \sigma_{0}\right)^{\prime \prime} \cos n \omega t=\sigma_{0} \sum_{n \text { odd }} \underbrace{n \omega J_{n}^{\prime \prime}\left(\omega, \sigma_{0}\right)}_{f_{n}} T_{n}(x)
\end{aligned}
$$

The above representation follows from the identity $T_{n}(\cos \theta)=\cos n \theta$. The resulting material coefficients in Eqns. 10 and 11 have units consistent with compliances $c_{n}\left(\omega, \sigma_{0}\right)[\mathrm{Pa}]^{-1}$ and fluidities $f_{n}\left(\omega, \sigma_{0}\right)[\mathrm{Pa} . \mathrm{s}]^{-1}$, respectively. Due to the convexity of the 3rd Chebyshev polynomial, we can infer that positive values of $c_{3}$ (i.e. increasing compliance) mean that the apparent elastic stress-strain curve shows stress-softening, while positive values of $f_{3}$ (i.e. increasing fluidity) imply stress-thinning of the apparent plastic strain-rate vs. shear stress 
curve. Conversely, negative values of $c_{3}$ imply stress-stiffening of the elastic material, while negative values of $f_{3}$ imply stress-thickening in the viscosity. In general, interpretations of these 3rd order Chebyshev coefficients can only be meaningful in the case when they are the leading order of nonlinearity. Furthermore, Klein et al. (2007); Ewoldt et al. (2010) and Rogers et al. (2011b) showed that in the limit of a perfectly plastic response, a $1 / n$ dependency is observed in the magnitude of the Fourier harmonics. Therefore, in the case of yielding materials, the Chebyshev coefficients with order $n \geq 5$ can play a significant role in determining the material response to LAOS. As a consequence, we will generally refrain from using the individual Chebyshev coefficients $f_{n}$ and $c_{n}$ to characterize material response, due to some of the concerns discussed by Rogers and Lettinga (2012). Instead, we define a set of general nonlinear measures that characterize significant features of the material response to LAOS at a particular temporal point within a sequence of cyclic deformations. These definitions are identical to those given by Ewoldt (2009) and Läuger and Stettin (2010); however, the use of a cosine in Eq. 6 (Ewoldt, 2009) instead of a sine (Läuger and Stettin, 2010) results in a slightly different expression for these measures in terms of the compliances $J_{n}^{\prime}$ and $J_{n}^{\prime \prime}$. Here the measures are also related to the LAOStress Chebyshev framework as well. The first of these measures is the minimum-stress elastic compliance, denoted here as $J_{M}^{\prime}$, which is defined and related to the compliance coefficients $J_{n}^{\prime}$ or $c_{n}$ as follows:

$$
\left.J_{M}^{\prime} \equiv \frac{d \gamma}{d \sigma}\right|_{\sigma=0}=\sum_{n \text { odd }}(-1)^{(n-1) / 2} n J_{n}^{\prime}=\sum_{n \text { odd }}(-1)^{(n-1) / 2} n c_{n}
$$

In Fig. 4 we illustrate the difference between the nonlinear measure $J_{M}^{\prime}$ for stresscontrolled LAOS and the complementary measure $G_{M}^{\prime}$ for strain-controlled LAOS, which is the minimum-strain elastic modulus (Ewoldt et al., 2008) defined as follows:

$$
G_{M}^{\prime}=\left.\frac{d \sigma}{d \gamma}\right|_{\gamma=0}
$$

In each figure, we show two sets of data - one Lissajous-Bowditch curve is measured for the Carbopol system undergoing stress-controlled LAOS $\left(\sigma=\sigma_{0} \cos \omega t\right)$, and the other Lissajous-Bowditch curve of the same material under strain-controlled LAOS $\left(\gamma=\gamma_{0} \sin \omega t\right)$. In the linear viscoelastic regime (at small strains and stresses) shown in Fig. 4 (a) the material response is indistinguishable in LAOStress or LAOStrain loading, as expected. In Fig. $4(\mathrm{~b})$ the values of $\gamma_{0}$ and $\sigma_{0}$ were chosen such that the two experiments would 

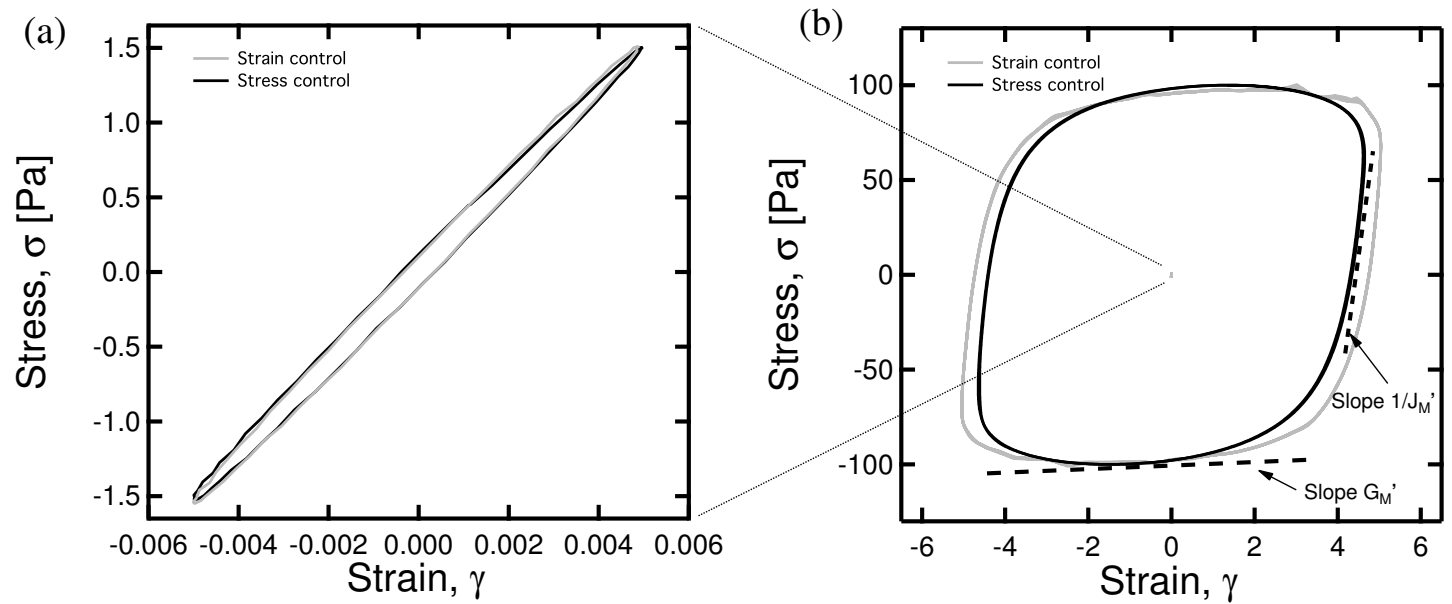

FIG. 4. (b) graphical comparison in the nonlinear (yielded) regime of the complementary measures $G_{M}^{\prime}$ and $J_{M}^{\prime}$ evaluated for stress-controlled LAOS (with $\sigma_{0}=100 \mathrm{~Pa}$ ) and strain-controlled LAOS (with $\gamma_{0}=5$ ) data from Carbopol at a frequency of $\omega=0.5 \mathrm{rad} / \mathrm{s}$. A comparison (a) in the linear viscoelastic regime of the Lissajous curves obtained in LAOStress and LAOStrain oscillatory tests.

result in approximately the same maximum stress and strain. It can be seen that the qualitative shape of the Lissajous curves is remarkably similar in both cases, even though the two experimental protocols are different. Specifically, in each curve we can identify an identical sequence of processes that the material undergoes; corresponding to elastic loading, followed by plastic flow and then elastic unloading. We see that the minimum stress elastic compliance $J_{M}^{\prime}$ measures the instantaneous compliance when the instantaneous imposed stress passes through zero, while $G_{M}^{\prime}$ measures the instantaneous modulus when the instantaneous imposed strain passes through zero. The critical difference between the two measures becomes apparent in their location on the Lissajous curve. We see that $J_{M}^{\prime}$ probes behavior in the elastic region of the curve (at stresses below the critical stress), while $G_{M}^{\prime}$ probes the behavior in the plastic flow region of the curve at stresses greater than the critical stress. The compliance $J_{M}^{\prime}$ is therefore a better indicator of the intracycle elastic behavior of the material in the unyielded region, and one of the main benefits of the stresscontrolled LAOS framework is that $J_{M}^{\prime}$ can be evaluated using the individual compliance coefficients $J_{n}^{\prime}$ obtained from the Fourier-Chebyshev spectrum (Eq. 12). This definition of $J_{M}^{\prime}$ corresponds to the reciprocal of the "Cage Modulus" defined and used by Rogers et al. (2011b), although Rogers et al. (2011b) based their definition of "Cage Modulus" on a strain-controlled experiment. The measure $J_{M}^{\prime}$ can, of course, be numerically evaluated for both stress and strain-controlled experiments, and from Fig. 4 (b) the two appear to have 
similar values (they are in fact within $7 \%$ of each other), however in the general nonlinear case they will not be equal due to the different loading history of the experiments.

These results suggest an alternative interpretation for the measure $G_{M}^{\prime}$ for yielding materials. Under a controlled oscillatory strain, $G_{M}^{\prime}$ is evaluated at zero instantaneous strain. However, the choice of this zero strain point is somewhat arbitrary, due to the fact that for a yielding material, irreversible viscoplastic flow is the dominant mechanism of deformation. Therefore, an alternative interpretation for $G_{M}^{\prime}$ is that it evaluates the material elasticity at maximum strain rate. As is observed in Fig. $4, G_{M}^{\prime}$ decreases dramatically past the yielding point, suggesting that in this material elasticity only plays a small role at maximum strain rate in the yielded regime of the Lissajous curve. However, care must be taken in using this interpretation to develop a constitutive model since, in general, descriptive rheological material functions are not necessarily prescriptive constitutive model parameters. In the upcoming sections we will discuss a model which captures this behavior by implementing a yielding element in series with a linear elastic element, and the decrease in the material function $G_{M}^{\prime}$ being caused by the onset of plastic flow in the material.

Another complementary nonlinear measure that can be defined is the elastic compliance $J_{L}^{\prime}$ at large stresses, which is related to the compliances $J_{n}^{\prime}$ and $c_{n}$ as follows:

$$
\left.J_{L}^{\prime} \equiv \frac{\gamma}{\sigma}\right|_{\sigma=\sigma_{0}}=\sum_{n \text { odd }} J_{n}^{\prime}=\sum_{n \text { odd }} c_{n}
$$

Both $J_{L}^{\prime}$ and $J_{M}^{\prime}$ are nonlinear elastic measures in the sense that they describe local intracycle elastic behavior in a nonlinear soft solid material or yield stress fluid. If desired, it is also possible to define a third nonlinear measure $J_{K}^{\prime}=d \gamma^{\prime} /\left.d \sigma\right|_{\sigma=\sigma_{0}}$ which is analogous to the measure $G_{k}^{\prime}$ introduced by Ewoldt et al. (2008). In addition to these measures, a relative ratio of the change in compliance within a large amplitude cycle can be defined:

$$
R \equiv \frac{J_{L}^{\prime}-J_{M}^{\prime}}{J_{L}^{\prime}}=\frac{4 c_{3}-4 c_{5}+8 c_{7}-8 c_{9}+\ldots}{c_{1}+c_{2}+c_{3}+\ldots}
$$

When $R>1$, the material exhibits pronounced nonlinearities in the form of an apparent softening.

It is also possible to define corresponding nonlinear viscous measures; for LAOStress these are based on the measured fluidities instead of compliances. The first of these is $\phi_{M}^{\prime}$, which 
TABLE I. Comparison of the nonlinear measures appropriate for stress-controlled LAOS with the analogous nonlinear measures for strain-controlled LAOS.

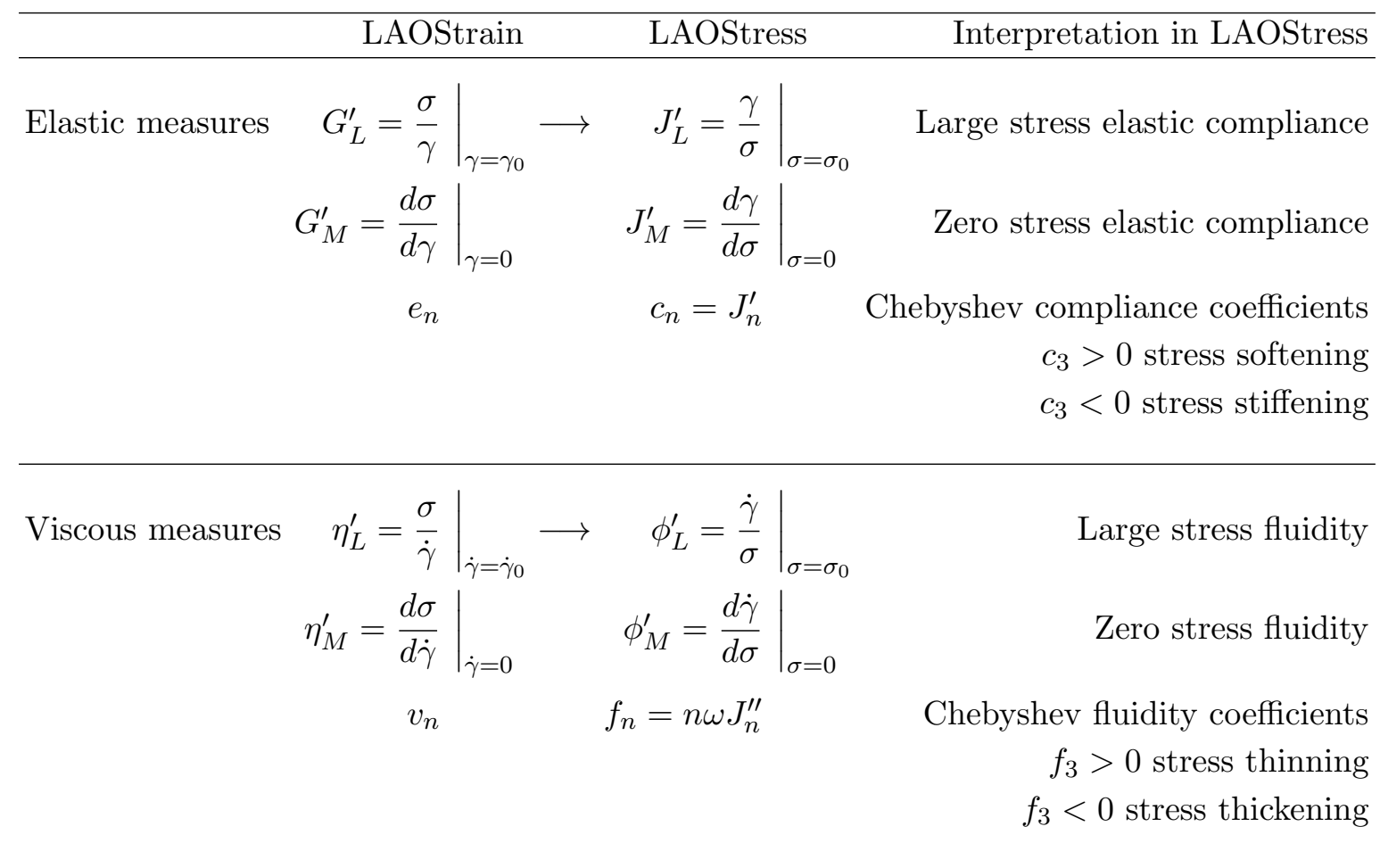

is the minimum stress fluidity and is defined as follows:

$$
\left.\phi_{M}^{\prime} \equiv \frac{d \dot{\gamma}}{d \sigma}\right|_{\sigma=0}=\sum_{n \text { odd }}(-1)^{(n-1) / 2} n^{2} \omega J_{n}^{\prime \prime}=\sum_{n \text { odd }}(-1)^{(n-1) / 2} n f_{n}
$$

The second is $\phi_{L}^{\prime}$, which is the fluidity at large stress and has the following definition:

$$
\left.\phi_{L}^{\prime} \equiv \frac{\dot{\gamma}}{\sigma}\right|_{\sigma=\sigma_{0}}=\sum_{n \text { odd }} n \omega J_{n}^{\prime \prime}=\sum_{n \text { odd }} f_{n}
$$

The nonlinear measures are summarized in the Table I, and are also contrasted with the analogous nonlinear measures defined for LAOStrain.

One important issue that bears mentioning is the interrelation between the measures defined under stress and strain-controlled oscillatory conditions. It is well known that in the linear regime of deformation, the dynamic compliances $J^{\prime}$ and $J^{\prime \prime}$ can be interrelated to the dynamic moduli $G^{\prime}$ and $G^{\prime \prime}$ (they are complex conjugates, but are not directly reciprocally related (Ferry, 1980)). One of the important aspects of the nonlinear relations defined 
in Table $\mathrm{I}$ is that because there is no general interrelation between the stress-dependent nonlinear compliances $J_{n}^{\prime}$ and nonlinear moduli $G_{n}^{\prime}$ for the strain-controlled case, there is no general interrelation between nonlinear measures such as $J_{M}^{\prime}$ and $G_{M}^{\prime}$. These measures are therefore complements, but not conjugates. Furthermore, the nonlinear LAOStress measures such as $J_{M}^{\prime}$ can actually be defined under either LAOStress or LAOStrain conditions, since their definitions are not based on a particular Fourier decomposition or mode of forcing. However they will not necessary yield the same value in both cases (see Fig. 4). Care must therefore be taken when reporting and interpreting different measures of nonlinear rheological response, and it is particularly important to keep track of whether these are evaluated under stress or strain-controlled conditions. In this work, whenever LAOStress measures such as $J_{M}^{\prime}, J_{L}^{\prime}, \phi_{M}^{\prime}$, and $\phi_{L}^{\prime}$ are referred to, it is implicit that they are evaluated for controlled-stress experiments (i.e. for $\sigma=\sigma_{0} \cos \omega t$ ). Despite this caveat, the experimental data in Fig. 4 for the particular case of the Carbopol microgel suggests that the evaluation of $J_{M}^{\prime}$ is not particularly sensitive to whether the experiment is done under LAOStress or LAOStrain (only a $7 \%$ change in the value is apparent at $\omega=0.5 \mathrm{rad} / \mathrm{s}$ and at $\sigma_{0}=100$ $\mathrm{Pa})$.

\section{RESULTS AND DISCUSSION}

\section{A. Rheology of an 'ideal' (non-thixotropic) material}

We now focus on a detailed illustration of the utility of the stress-controlled LAOS framework described in Sec. III by applying it to rheological fingerprinting of the Carbopol microgel. Before probing the behavior of the Carbopol system using LAOS, it is instructive to demonstrate some of the other important aspects of the rheology of this material. In Fig. 5 we show the steady state flow curve of the microgel in (a), and in (b) the linear viscoelastic moduli of the microgel are plotted as a function of frequency.

The steady flow data in Fig. 5 is obtained by imposing a given shear rate on the material, and then waiting approximately 3 minutes for the system to attain a steady state. The protocol is then repeated at a lower shear rate, and the shear rate is reduced progressively in steps to $\dot{\gamma}_{\min }=10^{-3} \mathrm{~s}^{-1}$. The data is fitted to the Herschel-Bulkley model, which is given 
(a)

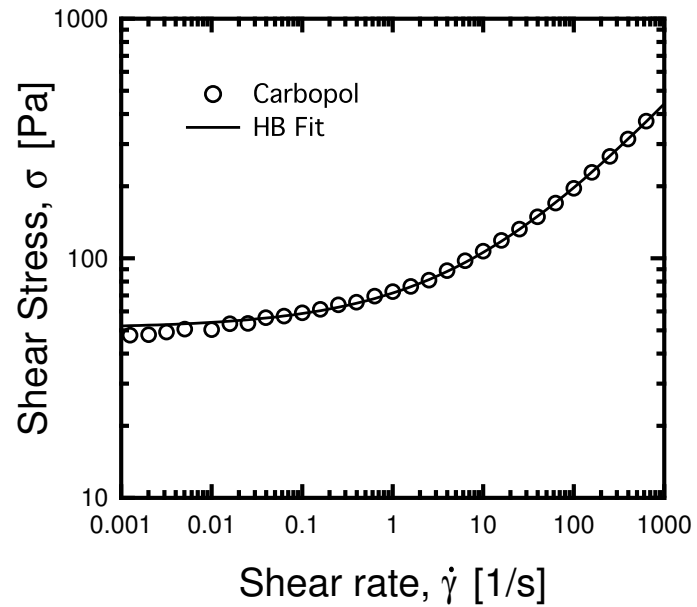

(b)

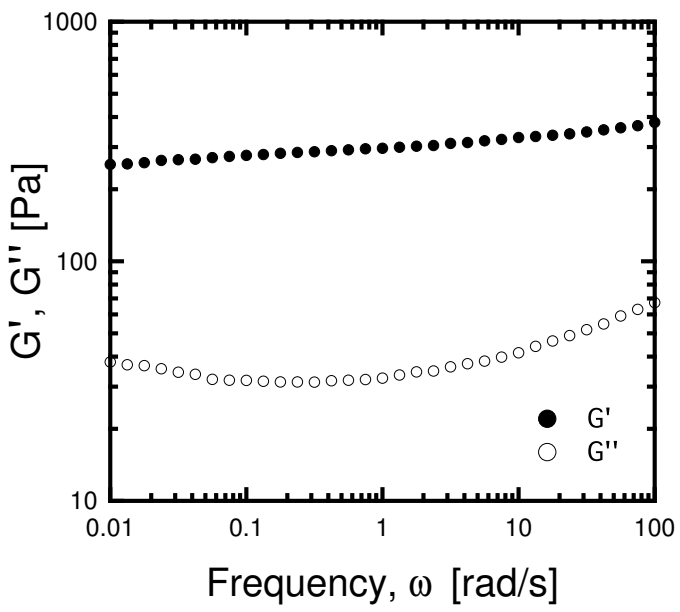

FIG. 5. Flow curve and linear viscoelastic moduli of the Carbopol microgel using roughened cone and plate fixtures. Linear viscoelastic moduli are obtained with a strain amplitude $\gamma_{0}=4 \%$, corresponding to a maximum stress of $\sigma_{\max } \simeq 15 \mathrm{~Pa}$, which is well below the dynamic yield stress of $\sigma_{y} \simeq 50 \mathrm{~Pa}$.

by the following expression:

$$
\sigma=\sigma_{y}+k \dot{\gamma}^{m}
$$

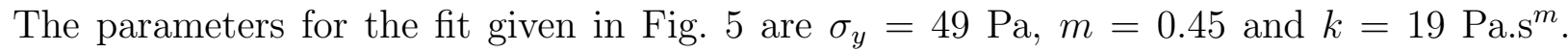
The Herschel-Bulkley model is well known to predict the steady state flow behavior of these types of microgels above the yield stress $\sigma_{y}$, and has been used in a large number of previous studies (Piau, 2007; Oppong and de Bruyn, 2007; Coussot et al., 2009). Fig. 5 shows that there is a good agreement between the HB fit and the data, with some deviation at the lower shear rates. The linear viscoelastic moduli show that the Carbopol gel behaves as a viscoelastic solid at low strain amplitudes, with $G^{\prime}$ generally about one order of magnitude larger than $G^{\prime \prime}$. Furthermore, there is a very weak power-law dependency of the parameter $G^{\prime}$ on frequency.

One of the interesting reported aspects of the rheology of Carbopol gels is the slow creeping flow observed at applied stresses $\sigma_{0}$ which are below the yield stress $\sigma_{y}$. This can be inferred by noting that (with the selected test protocol of 3 minutes shearing at each rate) the data points for the steady state flow curve progressively deviate slightly below the Herschel-Bulkley prediction in Fig. 5 as the stress approaches $\sigma_{y}$. This suggests that while the parameter $\sigma_{y}$ determined from Fig. 5 does correspond, in a limiting sense, to a "steady state" yield stress, the material is still able to creep viscoplastically below this yield stress. 
This type of creep has been documented by previous workers (Møller et al., 2009; Møller et al., 2009), who have shown that the measured instantaneous viscosity, $\eta^{+}(t) \equiv \sigma_{0} / \dot{\gamma}(t)$ during creep tests with an applied stress $\sigma_{0}<\sigma_{y}$ continues to increase in time, typically as a power-law with $\eta^{+}(t) \sim t^{n}$. Because the goal of our stress-controlled LAOS framework is to probe the behavior both above and below the steady state yield stress value $\sigma_{y}$, it is helpful to understand the nature of this slow viscoplastic creep occurring for stresses $\sigma<\sigma_{y} \simeq 49$ Pa. We present data from creep tests carried out on the Carbopol microgel in Fig. 6 below.
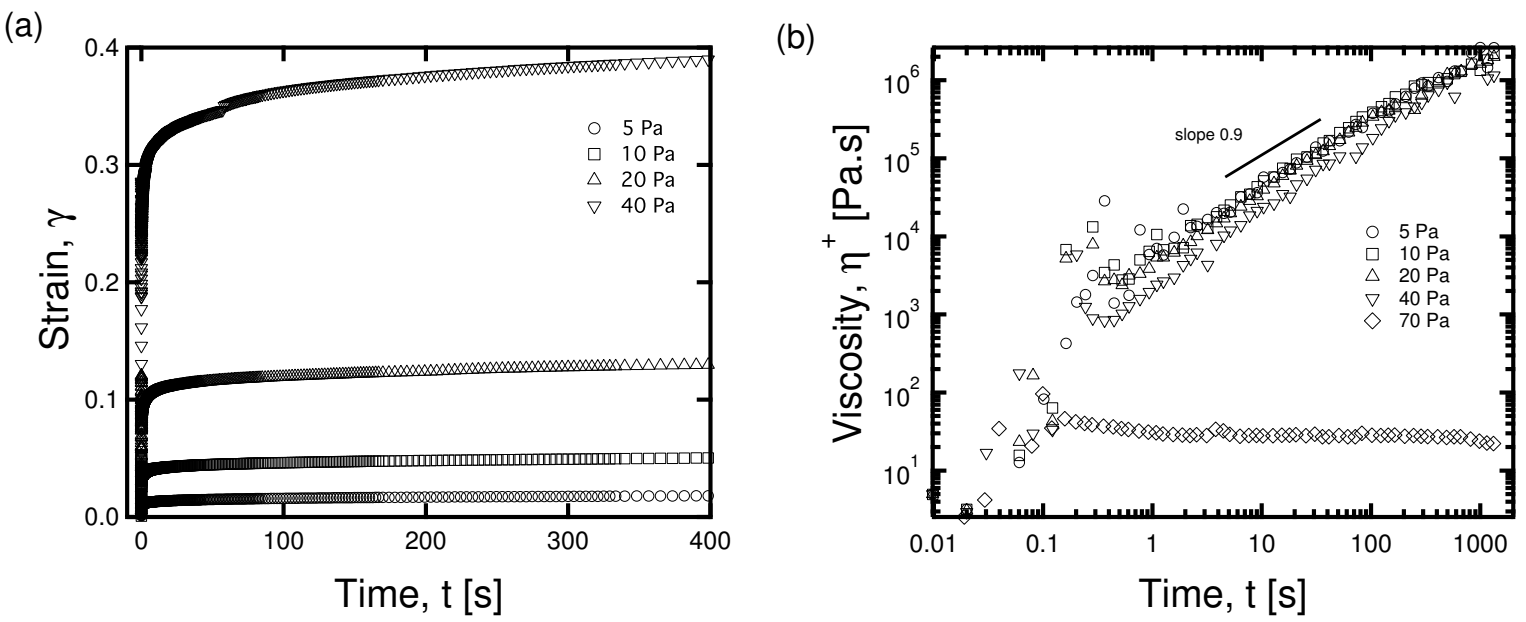

FIG. 6. Experimental creep data for the Carbopol system at a number of different applied stress values $\sigma_{0}<\sigma_{y}$. In (a) the measured strain $\gamma(t)$ vs. time is plotted, in (b) the instantaneous viscosity $\eta^{+}(t)$ is plotted vs. time.

The creep curves in Fig. 6 (a) are characterized by a rapid initial elastic strain response, followed by a long period of slow creep. In Fig. 6 (b) the instantaneous viscosity $\eta^{+}$as a function of time is plotted on a logarithmic scale - this viscosity is determined by dividing the imposed stress $\sigma_{0}$ by the local slope of the creep curve, i.e. the instantaneous shear rate $\dot{\gamma}(t) \equiv d \gamma / d t$. We see that when the applied stress is below $\sigma_{y}$, the long term creep behavior of this system is characterized by a power-law-like growth of the instantaneous viscosity with time (with viscosity scaling as $\eta^{+} \sim t^{0.9}$ ). By contrast at imposed stresses $\sigma>\sigma_{y}$ the viscosity rapidly settles to a steady value consistent with the steady flow curve shown in Fig. 6 (a). This is similar to what has been observed for the class of fluids that undergo a "viscosity bifurcation" (Coussot et al., 2002), and is identical to the type of behavior observed by Møller et al. (2009) (although with a different value of the power-law exponent). The power-law viscosity bifurcation at a critical stress is difficult to replicate in constitutive models. For 
example, the simple Kelvin-Voigt model for viscoelastic solids predicts that under creep loading, the strain will increase as $-e^{-t / \tau}$, with $\tau$ being a characteristic retardation time. As a result, the instantaneous viscosity $\eta^{+}(t)$ will increase exponentially in time at any value of the imposed stress. The model developed by Saramito (2009) (which incorporates a yielding transition at a critical stress) exhibits the same deficiency as the Kelvin-Voigt model, because it predicts Kelvin-Voigt viscoelastic deformation below the yield stress. What is desired is a constitutive model that predicts a well-defined flow curve at large stresses and a smooth transition to a power-law increase in the instantaneous viscosity $\eta^{+}(t)$. An example of such a model would be the "Soft Glassy Rheology" (SGR) model introduced by Sollich (1998) and refined by Fielding et al. (2000, 2009). In the forthcoming sections, however, we describe an alternative and relatively simple evolutionary constitutive model that can capture this viscosity bifurcation and power-law behavior below a critical stress.

\section{B. Probing nonlinear behavior using LAOStress}

LAOStress experiments provide an ideal test methodology for probing the rheological behavior of the Carbopol microgel above and below the yield stress $\sigma_{y}$. By varying the magnitude of the imposed stress amplitude $\sigma_{0}$, it is possible to observe how the material responds to deformations in the linear elastic regime and the ultimate viscoplastic flow regime. We begin by inspecting the cyclic stress-strain loading curves (or Bowditch-Lissajous curves) of the Carbopol undergoing an imposed oscillatory deformation with $\sigma=\sigma_{0} \cos \omega t$ with frequency $\omega=1 \mathrm{rad} / \mathrm{s}$. Fig. 7 below shows the response of the material at various values of the imposed stress amplitude $\sigma_{0}$ :

The Lissajous curves (and all Lissajous curves that follow) are now plotted with the shear stress on the abscissa because the controlled input into the system is stress, such that $\sigma=\sigma_{0} \cos \omega t$. In Fig. 7 (a) we see that the strains accumulated for $\sigma_{0}=200 \mathrm{~Pa}$ are very large due to the irreversible viscoplastic flow which occurs in the material (note that at $\sigma_{0}=200$ $\mathrm{Pa}$ we are well beyond the yield stress $\sigma_{y}$ shown in Fig. 5). In Fig. 7 (b) we expand the ordinate scale to focus on the response for smaller stress amplitudes, and see the beginning of a transition in the shape of the curves that occurs for stress amplitudes approaching $\sigma_{y}=49$ Pa. The individual curves for varying stress amplitudes are overlaid on the same plot in order to illustrate an important point - the value of $J_{M}^{\prime}$, i.e. the instantaneous compliance 

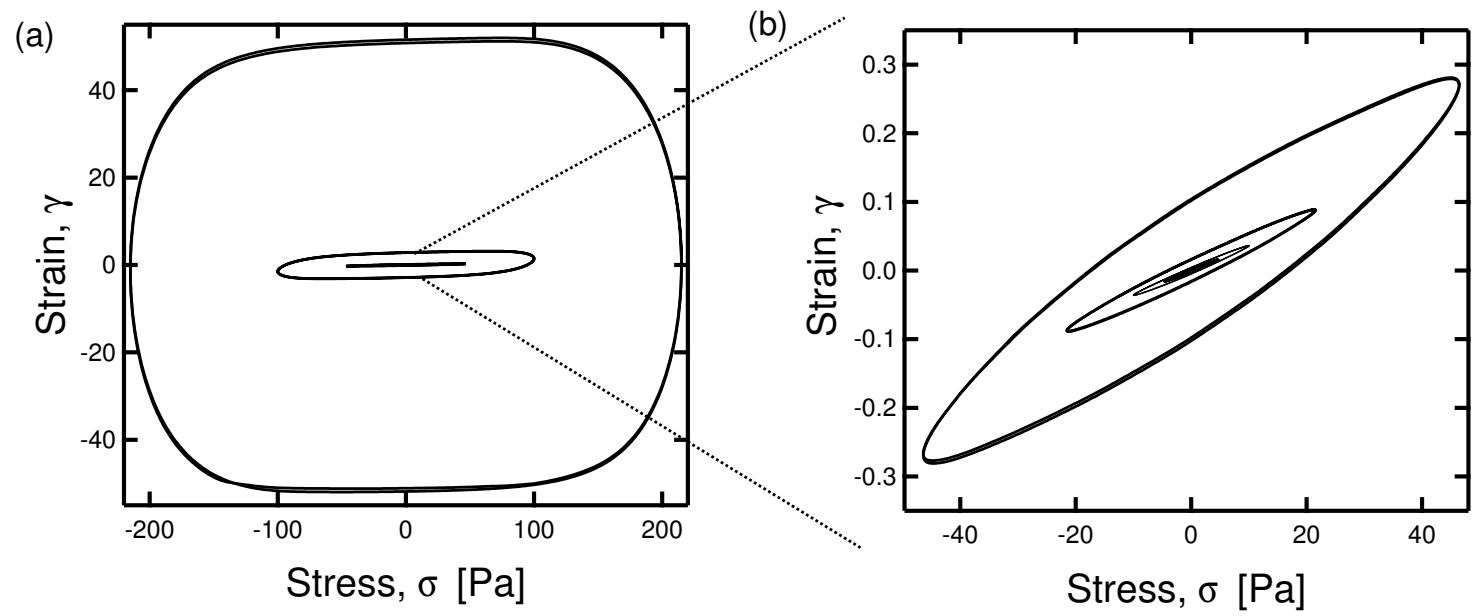

FIG. 7. LAOS of Carbopol at a number of different stress amplitudes $\sigma_{0}$ and a frequency of 1 $\mathrm{rad} / \mathrm{s}$. In (a) the Lissajous curves at the largest stress amplitudes are shown $\left(\sigma_{0}=200 \mathrm{~Pa}, 100\right.$ $\mathrm{Pa}$ ), smaller stress amplitude curves are shown on an expanded scale in (b)

at zero stress, systematically increases with $\sigma_{0}$, so that the material softens. However this nonlinear material measure does not vary as dramatically as $G_{M}^{\prime}$, which is the analogous strain-controlled LAOS measure defined in Eq. 13.

The full stress-amplitude/frequency dependence of the material response can be compactly illustrated through the use of a Pipkin diagram. However, a drawback to this graphical representation is that each cyclic curve has to be rescaled for clarity - this is potentially misleading because individual Lissajous curves may then appear to show large relative changes in the shape of their orbit as well as in associated local measures such as the compliance $J_{M}^{\prime}$. However, the Pipkin diagram is helpful for illustrating the fact that the yielding transition in this microgel is a gradual and frequency-independent one, i.e. based on the shape of the Lissajous curves, we cannot easily pick out a single stress amplitude at which we would uniquely identify that the material begins to flow. This is due to the dynamic nature of the stress-controlled LAOS protocol, and the tendency of Carbopol to exhibit some visco-plastic creep even at the lowest stresses $\sigma_{0} \ll \sigma_{y}$. As we show below, the Pipkin diagram also serves as a good construct for objectively comparing the experimentally measured response of the Carbopol to the predicted response of various constitutive models (i.e. it can serve as a fitting tool and discriminator).

In order to demonstrate that the small stress compliance $J_{M}^{\prime}$ exhibits a weak dependency on the stress amplitude $\sigma_{0}$ and frequency $\omega$ (a behavior that is not immediately apparent 


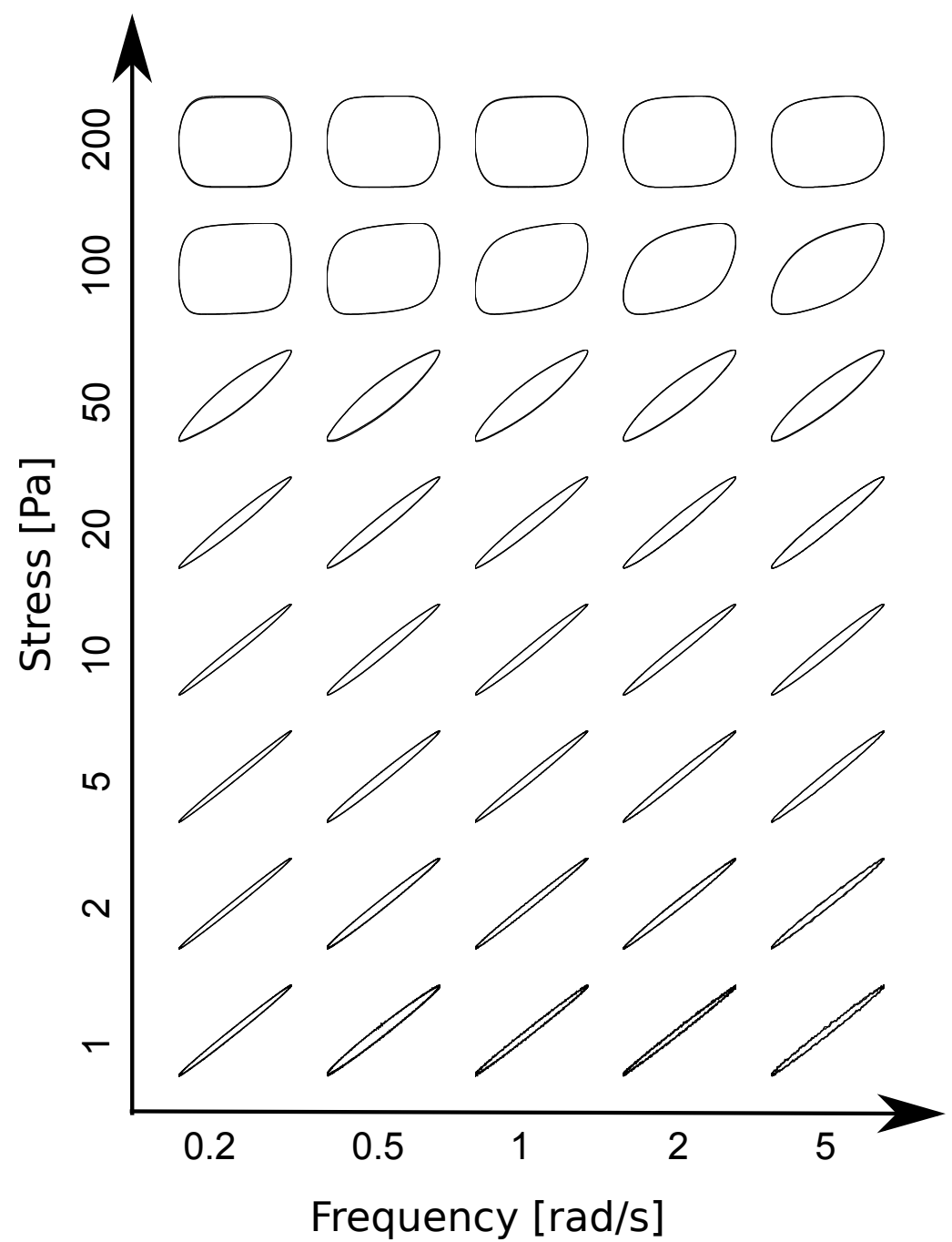

FIG. 8. Pipkin diagram of Carbopol response to LAOS at a number of different frequencies $\omega$ and stress amplitudes $\sigma_{0}$. At low stresses the material response shows little dependency on the frequency $\omega$. A stronger frequency dependence can be seen at the higher stresses.

from the Pipkin space plot, but is clearly seen from Fig. 7), we plot an interpolated contour plot of the minimum stress compliance $J_{M}^{\prime}\left(\sigma_{0}, \omega\right)$ which can be evaluated from Eq. 12 using the individual compliance coefficients $J_{n}^{\prime}$ determined for 5 different frequencies and 8 stress amplitudes. This contour plot is shown in Fig. 9 below, together with a corresponding contour plot of the nonlinear measure $J_{L}^{\prime}$ defined in Eq. 14 .

The contour plots of $J_{M}^{\prime}$ and $J_{L}^{\prime}$ provide a distinguishing fingerprint of the nonlinear material response. Firstly, we can see that $J_{M}^{\prime}$ exhibits a very weak dependency on frequency. It increases as the stress amplitude is increased (i.e. the material softens) and generally seems to reach a maximum at around $\sigma_{0}=100 \mathrm{~Pa}$, or approximately twice the critical yield stress 
(a)

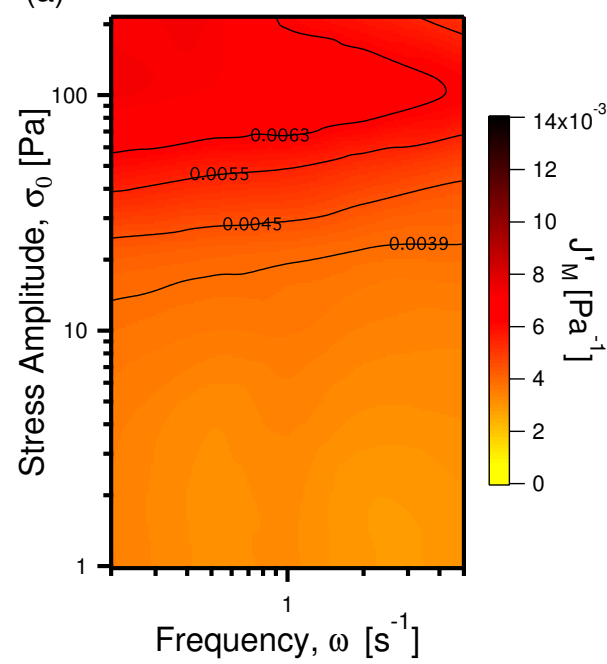

(b)

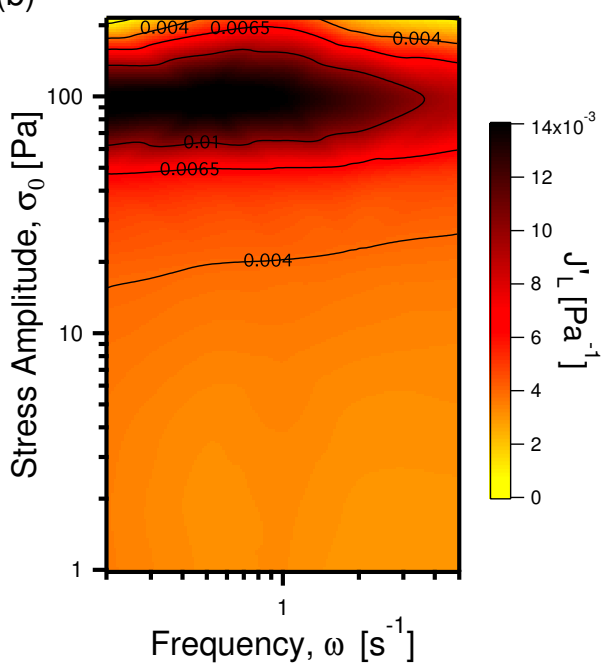

FIG. 9. Contour plots of the nonlinear compliance measures $J_{M}^{\prime}$ and $J_{L}^{\prime}$ (see Eq. 14 and Eq. 12). These measures show a weak frequency dependence, and in general increase as $\sigma_{0}$ is increased, reaching a maximum as the material begins to flow then subsequently decreasing.

identified from steady shear. However, the increase is moderate, raising the value of $J_{M}^{\prime}$ from $0.0039 \mathrm{~Pa}^{-1}$ at $\sigma_{0}=1 \mathrm{~Pa}$ to $0.0063 \mathrm{~Pa}^{-1}$ at $\sigma_{0}=100 \mathrm{~Pa}$. For small imposed stress amplitudes $\sigma_{0} \ll \sigma_{y}$, we see that the large stress compliance $J_{L}^{\prime}$ approaches the value of $J_{M}^{\prime}$ measured at zero instantaneous stress. This is to be expected because the material behaves primarily as a linear elastic solid for stresses $\sigma_{0} \ll \sigma_{y}$, and in the linear viscoelastic limit $J_{M}^{\prime}=J_{L}^{\prime}=J_{1}^{\prime}$.

It is also possible to create contour plots of the corresponding nonlinear viscous measures, $\phi_{L}^{\prime}$ and $\phi_{M}^{\prime}$ in order to gain further insight into the material response. These nonlinear measures of fluidity are presented in Fig. 10 and exhibit a somewhat less complicated dependence on $\omega$ and $\sigma_{0}$. In particular, $\phi_{L}^{\prime}$ is completely frequency independent. It is zero for small values of $\sigma_{0} \ll \sigma_{y}$ then exhibits a sudden increase beyond the yield stress $\sigma_{y}$ as the material begins to exhibit substantial plastic flow. For this class of materials, the fluidity $\phi_{L}^{\prime}$ therefore serves as a good indicator of the range of imposed stresses beyond which the material yields visco-plastically. Conversely, the fluidity measured at zero stress, $\phi_{M}^{\prime}$, exhibits a dependency on both $\omega$ and $\sigma_{0}$, but its relative changes are small compared to $\phi_{L}^{\prime}$. This initially unexpected dependency will be discussed in the context of two constitutive models in the upcoming sections.

One of the principal benefits of constructing rheological fingerprints such as the contour 


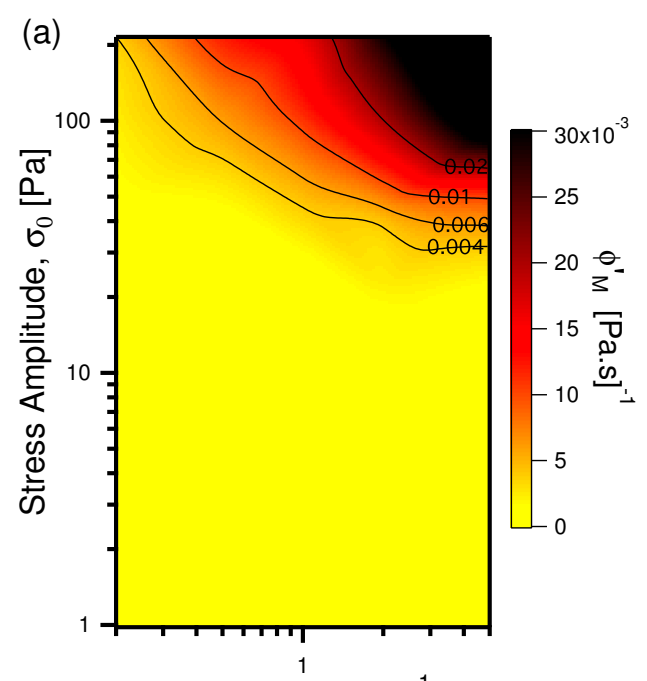

Frequency, $\omega\left[\mathrm{s}^{-1}\right]$

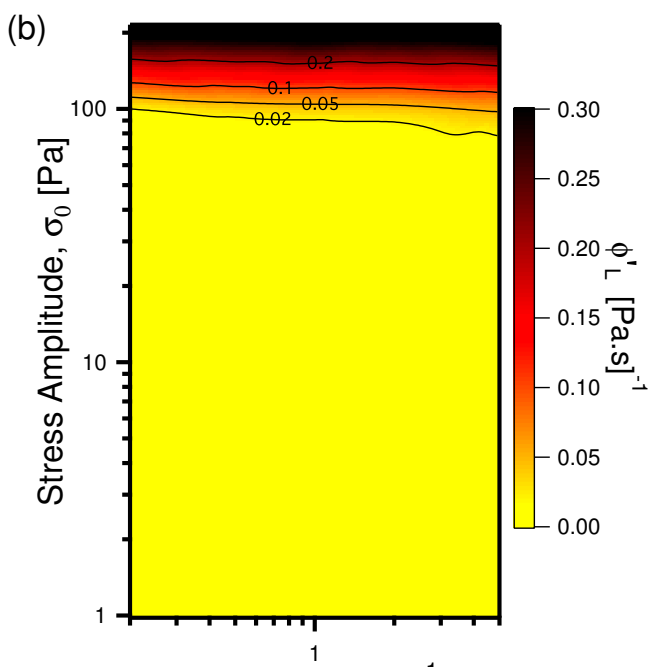

Frequency, $\omega\left[\mathrm{s}^{-1}\right]$

FIG. 10. Contour plots of the nonlinear fluidity measures $\phi_{M}^{\prime}$ and $\phi_{L}^{\prime} . \phi_{L}^{\prime}$ shows a clear increase as the stress amplitude is increased beyond the yield stress $\sigma_{y}$, indicating onset of yielding. A more complex behavior is exhibited by $\phi_{M}^{\prime}$, with both stress and frequency dependence.

plots presented in Figs. 9 and 10 is that they provide some insight into how to construct an appropriate constitutive model that captures the elasto-viscoplastic rheology of the Carbopol gel. A simple initial postulate is to model the Carbopol below the yield stress as an elastic solid with modulus $G$, with Herschel-Bulkley flow occurring as given in Eq. 18 above the yield stress. Such a proposition is initially reasonable, given the observation that both $J_{M}^{\prime}$ and $J_{L}^{\prime}$ are almost independent of $\omega$ and $\sigma_{0}$ below the yield stress. Furthermore, the very sudden transition in the fluidity $\phi_{L}^{\prime}$ measured at large stress amplitudes indicates that there is a distinct difference between the behavior exhibited above and below the critical stress $\sigma_{y}$. A piecewise continuous constitutive model is easy to develop, and is described in the next section. However we will show that there are several aspects of the measured Carbopol behavior that it does not capture. Specifically, it is unable to capture the stressdependent softening that is observed in the nonlinear material measures $J_{M}^{\prime}, J_{L}^{\prime}$ below the yield point. After noting these deficiencies, we then modify the constitutive model to capture the elastoplastic creep that is observed experimentally at small stresses. This "kinematic hardening" model captures the variation in the nonlinear compliances $J_{M}^{\prime}, J_{L}^{\prime}$, and fluidity $\phi_{M}^{\prime}$, and enables us to capture more completely the rheological fingerprint of this yielding microgel. 


\section{The Elastic Herschel-Bulkley Model}

For an elastic Herschel-Bulkley (EHB) material we begin in a manner similar to the approach taken for the canonical elastic-perfectly plastic constitutive model that was discussed in Sec. III A. The model developed here is discussed in the context of one-dimensional deformations, however a full three-dimensional version of this model is outlined in Appendix A.

First, the total strain is decomposed into elastic and plastic components such that $\gamma=$ $\gamma^{e}+\gamma^{p}$ as in Eq. 2. This strain decomposition is an essential component of this model, and the stress-controlled LAOS framework is more appropriate for analyzing the sequence of

physical processes envisioned by such a model. The stress $\sigma$ in the material is related to the elastic strain through the modulus $G$, i.e. $\sigma=G \gamma^{e}$ as in Eq. 3, and this elastic contribution to the total strain is retained beyond the yield point. The rate of plastic strain $\dot{\gamma}^{p}$ is then related through the following conditional equation:

$$
\dot{\gamma}^{p}= \begin{cases}0 & \text { if }|\sigma|<\sigma_{y} \\ n^{p}\left(\frac{|\sigma|-\sigma_{y}}{k}\right)^{1 / m} & \text { if }|\sigma| \geq \sigma_{y}\end{cases}
$$

Where the material constants $\sigma_{y}, m$ and $k$ are the same as those given in the simple HerschelBulkley model of Eq. 18, and $n^{p}$ is the direction of stress, i.e. $n^{p}=\sigma /|\sigma|$. The presence of the directional integer $n^{p}(= \pm 1)$ forces the plastic strain rate to be co-directional with the imposed shear stress. The only addition to the simple viscoplastic Herschel-Bulkley model of Eq. 18 is that elastic behavior has been introduced (and this elastic behavior is present both above and below the yield stress). Compared to the simplest elastic-perfectly plastic material discussed in Section III A, we have introduced a well-defined rate dependency in the plastic flow rule given by Eq. 19. One of the important differences between this particular model and some of the Elastic-Bingham (Yoshimura and Prud'homme, 1987) or Elastic HerschelBulkley (Doraiswamy et al., 1991) type models used in the rheology literature is that there is no critical strain above which plastic flow begins for this model. The elastic strain $\gamma^{e}$ therefore does not saturate for this EHB model. This avoids the problems associated with having discontinuities in the stress that arise during strain-controlled oscillatory experiments, such as those shown by Yoshimura and Prud'homme (1987). Nevertheless, one downside of this EHB model that is immediately apparent from inspecting the flow rule in Eq. 19 is that for stresses below the yield stress value $\sigma_{y}$, the model will show no irreversible deformation or 
energy dissipation. This dissipation is a result of slow viscoplastic creeping behavior which is clearly exhibited by the Carbopol microgel even below the critical stress.

One way to inspect the overall ability of a model to predict the rheology of the Carbopol gel is to overlay the experimental measurements and predicted response of this model in the Pipkin space represented by Fig. 8. The resulting fingerprint illustrates many different aspects of the microgel's response to deformation, from elastic behavior at low stresses to fully viscoplastic yielding behavior at the higher stresses. In Fig. 11 we compare the LAOStress measurements with the response of the EHB model with fitting parameters $\sigma_{y}=$ $45 \mathrm{~Pa}, m=0.43, k=26 \mathrm{~Pa} . \mathrm{s}^{m}, G=350 \mathrm{~Pa}$. Note that these parameters are close to those obtained from fitting the steady flow curve in Fig. 5 (a), however they have been adjusted slightly in order to improve the fit of the EHB model to the experimental measurements. We also note that the elastic modulus $G$ is approximately equal to the elastic modulus $G^{\prime}$ measured in Fig. 5 (b).

From close inspection of the predictions in Fig. 11 it becomes apparent that the EHB model is successful at capturing the behavior of the Carbopol microgel under LAOS in two limiting regions. Firstly at large stress amplitudes where $\sigma_{0} \gg \sigma_{y}$ (e.g. $\sigma_{0}=200 \mathrm{~Pa}$ ). In this region the response of the material is primarily dictated by the Herschel-Bulkley flow rule in Eq. 18. The model also fits the data well at very low stresses $\sigma_{0} \ll \sigma_{y}$ (e.g. $\left.\sigma_{0}=1 \mathrm{~Pa}\right)$. At these low stresses the deformation of the Carbopol gel is close to that of a simple Hookean solid. The primary limitation to this EHB model, however, is that it is unable to capture the gradual increase in the hysteresis exhibited by the Lissajous curves as the imposed stress amplitude approaches the critical stress $\sigma_{y}$. This is because the model assumes perfectly elastic behavior below $\sigma_{y}$. By inspecting Fig. 6 or 7, we can see that Carbopol gel is far from a perfect linear elastic solid when $\sigma<\sigma_{y}$.

Plotting the nonlinear compliances $J_{M}^{\prime}$ and $J_{L}^{\prime}$ at a given frequency as the stress amplitude is incremented provides further insight into the deficiencies of the EHB model in capturing the nonlinear behavior of the Carbopol gel. In Fig. 12 we overlay onto the experimental data the values of these nonlinear measures for two models, the EHB model which has been discussed in this section (broken line), and the KH model (Kinematic Hardening model; solid line) which will be described in the next section.

It is clear that both the small strain and large strain compliances $J_{M}^{\prime}$ and $J_{L}^{\prime}$ increase as the stress amplitude $\sigma_{0}$ is increased and the material softens. Both values reach a maximum 


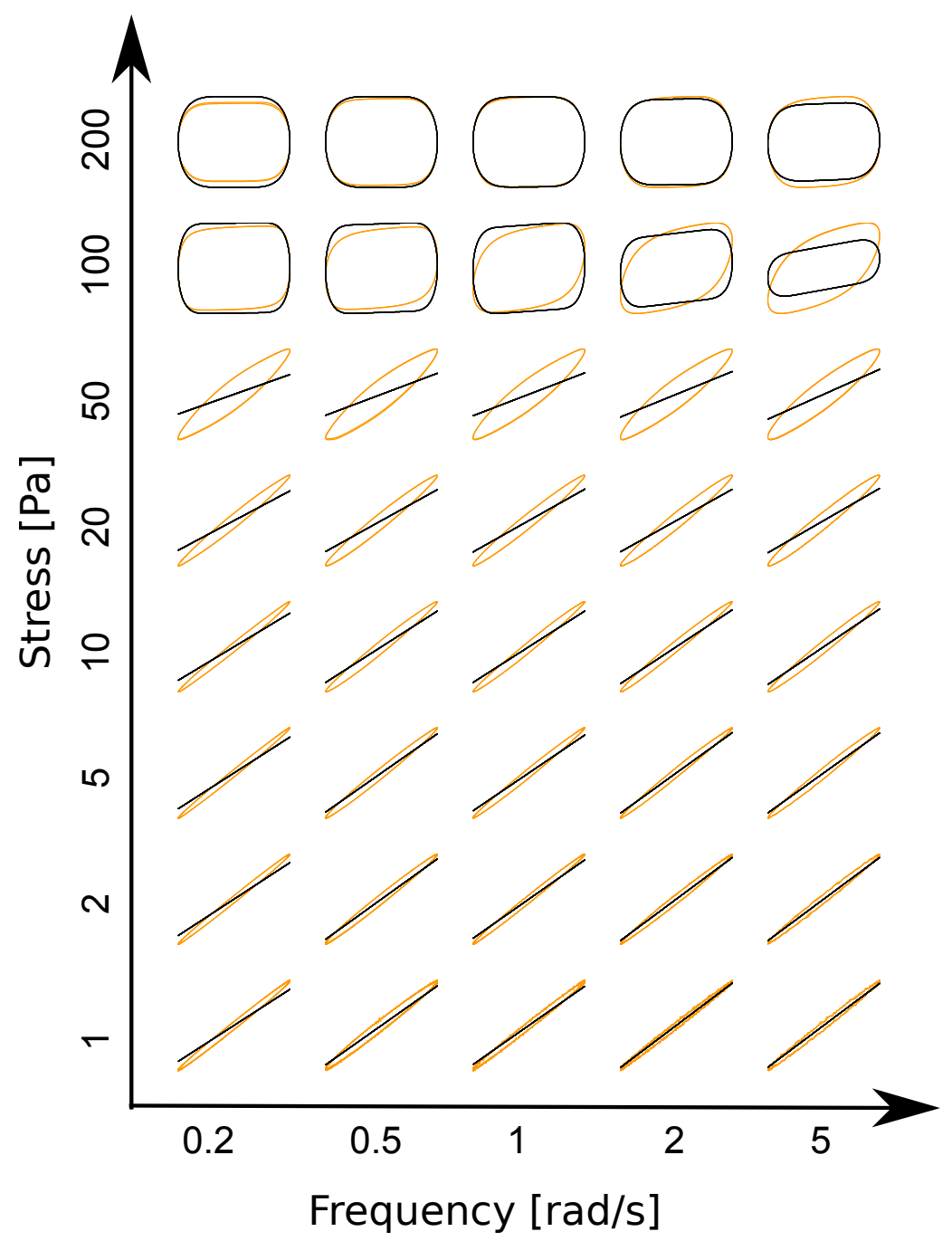

FIG. 11. Pipkin diagram showing comparison of EHB model (black) with Carbopol data (orange) at a number of different frequencies $\omega$ and stress amplitudes $\sigma_{0}$. Shown are individual Lissajous curves of strain $\gamma(t)$ vs. stress $\sigma(t)$. The EHB model fitting parameters are $\sigma_{y}=45 \mathrm{~Pa}, k=26$ Pa.s ${ }^{m}, G=350 \mathrm{~Pa}, m=0.43$.

at a value of $\sigma_{0} \simeq 100 \mathrm{~Pa}$, followed by a subsequent decrease in their value. The EHB model however, predicts an ideal elastic response with $J_{M}^{\prime}=J_{L}^{\prime}=1 / G=0.0029 \mathrm{~Pa}^{-1}$. The reason why this is the case can be easily understood if one considers the nature of the strain decomposition given in Eq. 8 and 9. We stated previously in Sec. III (and show in Appendix C) that the strain decomposition in Eq. 8 and 9 is unique in the sense that no other decomposition provides an apparent elastic strain $\gamma^{\prime}$ that is a single-valued function of $\sigma$, and an apparent plastic strain rate $\dot{\gamma}^{\prime \prime}$ that is a single-valued function of $\sigma$. For the EHB model, $\gamma^{e}$ is a single-valued function of $\sigma$ (defined through Eq. 3), and Eq. 19 is formulated such that $\dot{\gamma}^{p}$ is also a single-valued function of $\sigma$. It therefore follows that when 
(a)

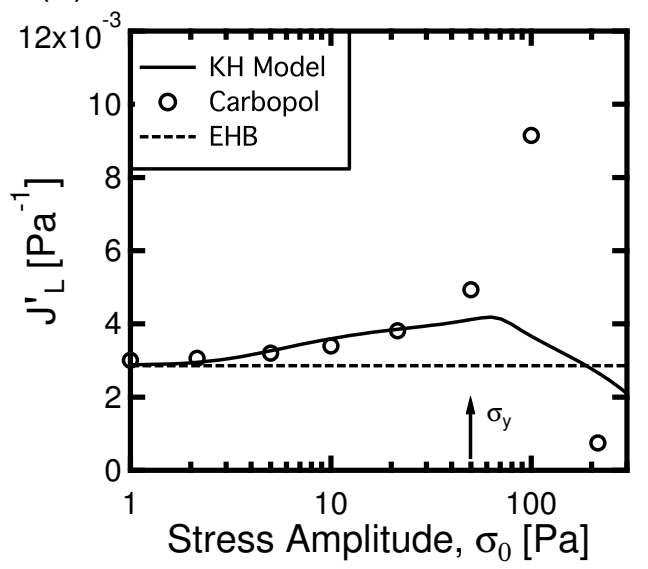

(b)

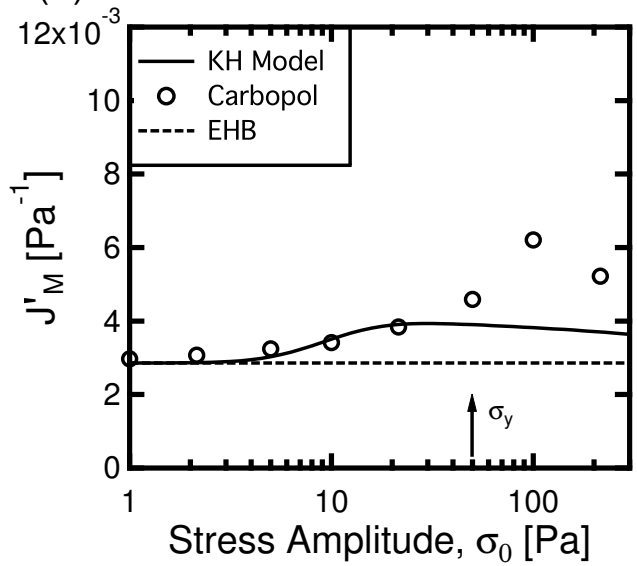

FIG. 12. Plots of the compliance measures $J_{M}^{\prime}$ and $J_{L}^{\prime}$ at a frequency of $\omega=5 \mathrm{rad} / \mathrm{s}$ for a range of stress amplitudes. Carbopol data is contrasted to two models, the Elastic Herschel-Bulkley model (fitting parameters $\sigma_{y}=45 \mathrm{~Pa}, k=26 \mathrm{~Pa} . \mathrm{s}^{m}, G=350 \mathrm{~Pa}, m=0.43$ ) and the Kinematic Hardening model $(\mathrm{KH})$ described in the next section.

the strain decomposition given in Eq. 9 is carried out for the EHB model undergoing a stress-controlled oscillatory deformation, we obtain $\gamma^{\prime}=\gamma^{e}$ and $\dot{\gamma}^{\prime \prime}=\dot{\gamma}^{p}$. Thus, for the EHB, model the unique value of elastic strain determined from the experimental strain decomposition of Eq. 9 is identical to the elastic strain defined in the constitutive model (Eq. 2). The nonlinear measure $J_{M}^{\prime}$ can then be determined by combining the definition in Eq. 12 with our strain decomposition of Eq. 8 and 9. Specifically:

$$
J_{M}^{\prime}=\left.\frac{d \gamma^{\prime}}{d \sigma}\right|_{\sigma=0}+\left.\frac{d \gamma^{\prime \prime}}{d \sigma}\right|_{\sigma=0}
$$

Using the chain rule and the fact that there are only odd harmonics present in the response of the EHB model, together with the fact that $\sigma=\sigma_{0} \cos \omega t$, it is possible to show that the second term in Eq. 20 is zero. Then, because $\gamma^{\prime}=\gamma^{e}$, the equation above is rewritten:

$$
J_{M}^{\prime}=\left.\frac{d \gamma^{e}}{d \sigma}\right|_{\sigma=0}=\left.\frac{d}{d \sigma} \frac{\sigma}{G}\right|_{\sigma=0}=\frac{1}{G}
$$

Similar reasoning can be used to show that $J_{L}^{\prime}=1 / G$ for all values of $\omega$ and $\sigma_{0}$ for the EHB model. In particular:

$$
J_{L}^{\prime}=\left.\frac{\gamma}{\sigma}\right|_{\sigma=\sigma_{0}}=\left.\frac{\gamma}{\sigma}\right|_{t=0}
$$


And because $\gamma^{\prime \prime}=\gamma^{p}$ is zero at $t=0$ we have:

$$
J_{L}^{\prime}=\left.\frac{\gamma^{e}}{\sigma}\right|_{t=0}=\frac{1}{G}
$$

It is therefore clear that although the EHB model is successful in capturing the behavior of the system at both very low stress amplitudes and very large stress amplitudes, the material response at intermediate stresses is more complex.

Similar arguments about the deficiencies of the EHB model can be made by inspecting plots of the nonlinear viscous measures $\phi_{L}^{\prime}$ and $\phi_{M}^{\prime}$ as shown in Fig. 13. The EHB model provides a very good agreement with experimental data for the fluidity measure $\phi_{L}^{\prime}$ - this is because $\phi_{L}^{\prime}$ is a measure of the viscous behavior of the material at large stresses, and this is dominated by the Herschel-Bulkley flow rule in Eq. 18. However, at small imposed stresses, the EHB model is purely elastic (no dissipation) and therefore predicts that for all values of $\omega$ and $\sigma_{0}$, the fluidity $\phi_{M}^{\prime}$ is identically zero. This follows from the definition of $\phi_{M}^{\prime}$, which samples the material response instantaneously at the point within an oscillatory cycle where the imposed stress is zero. The Carbopol gel, on the other hand, exhibits a progressive increase in $\phi_{M}^{\prime}$ as $\sigma_{0}$ is increased.

(a)

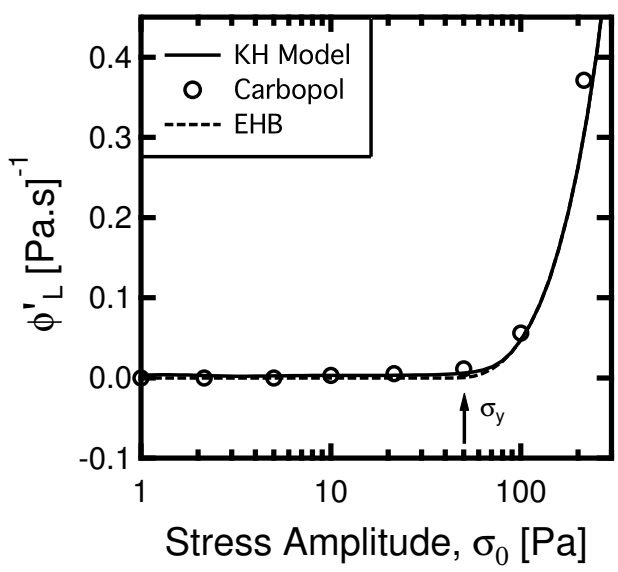

(b)

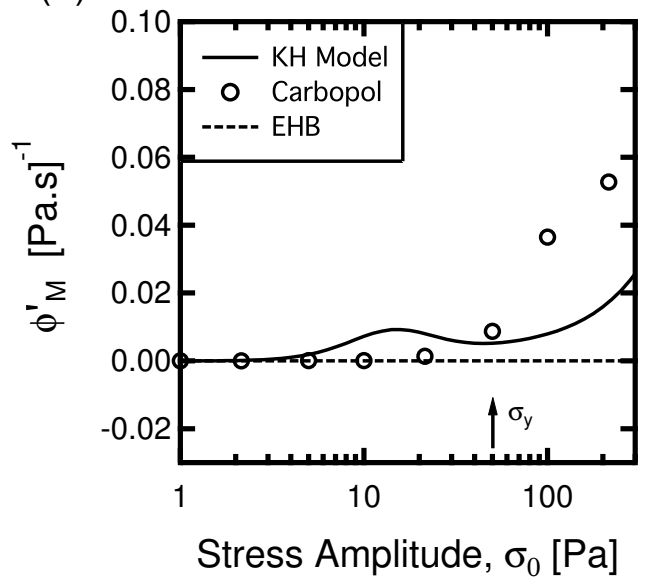

FIG. 13. Plots of the nonlinear fluidities $\phi_{M}^{\prime}$ and $\phi_{L}^{\prime}$ (note the difference in ordinate scales) at a frequency of $\omega=5 \mathrm{rad} / \mathrm{s}$ for a range of stress amplitudes. Carbopol data is contrasted to two models, the Elastic Herschel-Bulkley model (fitting parameters $\sigma_{y}=45 \mathrm{~Pa}, k=26 \mathrm{~Pa} . \mathrm{s}^{m}, G=350$ $\mathrm{Pa}, m=0.43)$ and the Kinematic Hardening model $(\mathrm{KH})$ described in the next section.

In order to motivate the development of a more advanced constitutive model, we have overlaid on Fig. 12 and 13 the predicted values of the nonlinear measures $J_{L}^{\prime}, J_{M}^{\prime}, \phi_{L}^{\prime}$ and 
$\phi_{M}^{\prime}$ for an alternate constitutive model which incorporates a behavior known as kinematic hardening (Lemaitre and Chaboche, 1990). The nonlinear response of this particular model appears to capture more completely the increase and subsequent decrease in the compliances $J_{L}^{\prime}$ and $J_{M}^{\prime}$ as the stress amplitude $\sigma_{0}$ is increased. In the following section we will show that by incorporating this behavior (which involves the addition of only one more material constant) we can capture the salient features of the Carbopol gel response to LAOStress deformations. This model also removes the somewhat arbitrary discontinuous flow/no flow condition given by Eq. 19. This more sophisticated kinematic hardening model will also test the strengths and limitations of the LAOStress strain decomposition (Eqns. 8 and 9) demonstrating a case in which the one-to-one equivalence between the experimentallymeasured strains defined in the decomposition of Eq. 8 \& 9, and the elastic and plastic strains, $\gamma^{e}$ and $\gamma^{p}$ in the model, breaks down.

\section{An Elasto-Plastic Material with Kinematic Hardening (KH Model)}

We seek to develop a constitutive model that improves on the ability of the EHB model to capture the rheological fingerprint of the Carbopol gel under LAOS. While there are many potential ways to accomplish this, there were two main criteria that were used to identify an appropriate model. The first is simplicity, i.e. the desire to introduce as few new additional parameters as possible to the existing EHB model. The second criterion was to build on well-established concepts that already exist in the plasticity literature.

Based on these criteria, the EHB model was modified in order to account for a behavior known as kinematic hardening. The basic concept behind the behavior of kinematic hardening is that it accounts for movement of the center of location of the yield surface (in stress space) for a given material (Lemaitre and Chaboche, 1990). For the simple 1-dimensional model which we will deal with here, this implies that the yield stress of the material evolves dynamically with time, as the material is deformed. Kinematic hardening is a concept that is widely used in the plasticity literature, but for the rheologist who is unfamiliar with the topic we recommend one of the many textbooks which have been written on the topics of continuum mechanics or plasticity (Lemaitre and Chaboche, 1990; Khan and Huang, 1995; Gurtin et al., 2010). For clarity, we have tried to keep the nomenclature in the present work consistent with that used in the plasticity and solid mechanics literature (particularly 
with that of Gurtin et al. (2010), Henann and Anand (2009), Anand et al. (2008) and Ames et al. (2009)). While the case discussed in detail here is a simple 1-dimensional version, in Appendix A we outline the formulation of a frame-invariant, thermodynamically-consistent version of the constitutive model in $3 \mathrm{D}$ tensorial form. The reader can also consult Henann and Anand (2009) for a complete version of the model with combined kinematic and isotropic hardening.

As was the case for the EHB model considered in Section IV C, the strain in the KH model admits an additive decomposition; $\gamma=\gamma^{e}+\gamma^{p}$ as in Eq. 2. We follow the approach of Gurtin et al. (2010); first a simple form of the free energy of the material is proposed, from which equations for the stress follow. For this particular model, the defining equation for the free energy $\psi$ of the material is as follows:

$$
\psi=\frac{1}{2} G\left(\gamma^{e}\right)^{2}+\frac{1}{2} C(A)^{2}
$$

The free energy contains two terms, the first is an elastic free energy, which depends on the elastic strain $\gamma^{e}$. This first term would be equivalent for the simpler EHB model, but for the KH model we introduce a second term, a "defect" energy which depends on a dimensionless internal variable $A$. In the context of polycrystalline metals, this defect energy would be the result of the formation and movement of dislocations in the crystal lattice. For the Carbopol system, the defect energy results from corresponding features relevant to the specific microstructure of the material. Piau (2007) described the microstructure of Carbopol gels as consisting of individual swollen polymeric sponges. At a high enough volume fractions, these constituent elements likely become trapped in "cages" that are formed by the neighboring particles, and a defect energy may arise from slip occurring between these particles and a topological rearrangement of their structure (Menut et al., 2012). In Eq. 24 the variable $A$ is not a strain per se, but in the 3-dimensional generalization of this model (which is discussed in Appendix. A) it shares some of the properties of a finite strain tensor. In particular it was shown by Henann and Anand (2009) that the 3-dimensional tensorial version of this variable, $\mathbf{A}$, is equivalent to an "energetic" plastic left Cauchy-Green tensor (or Finger tensor). The defect variable $A$ evolves as the accumulated plastic strain $\gamma^{p}$ in the material varies. The parameter $C$ is a new material constant, termed the back stress modulus (Ames et al., 2009), which has dimensions of stress. 
From the form of the free energy that is specified in Eq. 24, the equation for the stress in the material is $\sigma=\frac{\partial \psi}{\partial \gamma^{e}}=G \gamma^{e}$ as in Eq. 3. While $\sigma$ is still the stress in the material, and corresponds to the stress that would be measured in an experiment, the presence of the additional term gives rise to a second contribution to the stress, referred to as the back stress, $\sigma_{\text {back }}=\frac{\partial \psi}{\partial A}$ :

$$
\sigma_{\text {back }}=C A
$$

The back stress corresponds to the center of the location of the yield surface (in stress space) in the material, and is integral to the development of this model. The fact that the value of the back stress varies with $A$, allows for time variation in the "effective" stress, $\left|\sigma-\sigma_{\text {back }}\right|$ within the material, which determines the plastic flow rate, even when the imposed stress $\sigma$ is held constant. The plastic flow rate $\dot{\gamma}^{p}$ is related to the effective stress through the following equation:

$$
\begin{aligned}
& \dot{\gamma}^{p}=n^{p}\left|\dot{\gamma}^{p}\right| \quad \text { where } \quad n^{p}=\frac{\sigma-\sigma_{\text {back }}}{\left|\sigma-\sigma_{\text {back }}\right|} \quad \text { (Direction of plastic flow) } \\
& \left|\dot{\gamma}^{p}\right|=\left(\frac{\left|\sigma-\sigma_{\text {back }}\right|}{k}\right)^{1 / m} \quad \text { (Magnitude of plastic flow rate) }
\end{aligned}
$$

The equation for the plastic flow rate for this particular model is slightly different from that of the EHB model, which is given in Eq. 19. The yield stress parameter $\sigma_{y}$ has been set to zero in the equation above, and the stress $|\sigma|$ has been replaced by the effective stress within the material that is driving plastic flow, $\left|\sigma-\sigma_{\text {back }}\right|$. We include the variable $n^{p}$ again in order to make it clear that the plastic flow is co-directional with the effective stress, and not with the total applied stress $\sigma$. The last important element of this constitutive model is to specify the evolution in the defect energy which involves the plastic flow rate $\dot{\gamma}^{p}$. The differential equation for the parameter $A$ in a simple one-dimensional form is as follows:

$$
\dot{A}=\dot{\gamma}^{p}-q A\left|\dot{\gamma}^{p}\right|
$$

This evolution equation accounts for so-called nonlinear kinematic hardening (due to the dependence of $\left|\dot{\gamma}^{p}\right|$ on $A$ in Eq. 27) and was first introduced by Armstrong and Frederick (1966) in order to account for the multiaxial Bauschinger effect. The differential equation is always evaluated with initial conditions of $A(0)=0$, i.e. the material is in a virgin state 
with zero defect energy at the start of the deformation. In the differential equation above (Eq. 28), we have introduced the dimensionless parameter $q$, as an additional material constant. Note that when compared to the EHB model, we have removed the material constant $\sigma_{y}$ and introduced two new constants, $q$ and $C$. We have therefore increased the number of material constants in this model relative to the EHB model by one.

It is now clear that with the addition of Eq. 28 to the constitutive model (given by Eqns. 24, 25, 26 and 27) we can have a dynamic evolution of the back stress $\sigma_{\text {back }}$ as the material is deformed. The implications of this evolution equation for $A$ on the flow behavior can be understood with some simple reasoning. The material always starts out with an initial value of $A(0)=0$. Then, if we consider the application of a constant stress $\sigma$, the material will initially deform elastically, but will also begin to experience some plastic flow due to the fact that the back stress is zero (Eq. 25) for the specified initial conditions. As plastic flow occurs, $\dot{A}$ will initially be positive, resulting in growth of the value of $A$. Eventually the second term in the differential equation in Eq. 28 will grow resulting in a reduction in the rate of change of $A$, until ultimately the material reaches a steady state flow corresponding to $\dot{A}=0$. Thus, the increase of $A$ with time results in a decrease in the rate of plastic flow $\dot{\gamma}^{p}$, or an apparent "kinematic hardening" which is exactly what is observed for creep tests of the carbopol system (see the creep curves in Fig. 6).

At steady state, the KH model approaches the same form as the EHB model, predicting Herschel-Bulkley flow behavior, which is also exhibited by the Carbopol gel as shown in Fig. 5. To show this, we set $\dot{A}=0$; from Eq. 28 it then follows that $q A= \pm 1$ (depending on the direction of the applied stress). Furthermore, from Eq. 25 it follows that $\sigma_{\text {back }}= \pm C / q$ at steady state. When we insert this steady state value of $\sigma_{\text {back }}$ into the flow rule of Eq. 27, we exactly recover the Herschel-Bulkley flow equation of Eq. 18 at steady state, and we identify $C / q=\sigma_{y}$. The ratio of material constants $C / q$ can therefore be determined by measuring a steady state flow curve such as the one presented in Fig. 5.

The KH model thus successfully captures the steady state flow behavior of the carbopol microgel at stress values $\sigma>\sigma_{y}$. It is also possible to show that the model successfully captures the correct creep behavior for imposed stresses below the yield stress. To show this, we plot in Fig. 14 the evolution of the apparent viscosity of this particular model, defined as $\eta^{+} \equiv \sigma_{0} / \dot{\gamma}(t)$, for a number of creep tests with different applied stresses $\sigma_{0}$. The model was simulated using an Euler forward-time integration scheme, with parameters set 
as follows: $G=350 \mathrm{~Pa}, C=540 \mathrm{~Pa}, q=12, k=26 \mathrm{~Pa} . \mathrm{s}^{m}, m=0.43$. Note that these are the same parameters as those used for the EHB fit in Fig. 11, however the value $\sigma_{y}=45$ $\mathrm{Pa}$ is now represented by the ratio $C / q$ leaving only one free parameter to adjust (either the value of $q$ or $C$ ). These values are also the same parameters that were used to evaluate the nonlinear compliances $J_{L}^{\prime}, J_{M}^{\prime}, \phi_{L}^{\prime}$ and $\phi_{M}^{\prime}$ for the kinematic hardening model shown in Fig. 12 and 13. The numerical values were determined to best capture the overall response of the Carbopol microgel to stress-controlled LAOS (see details below).

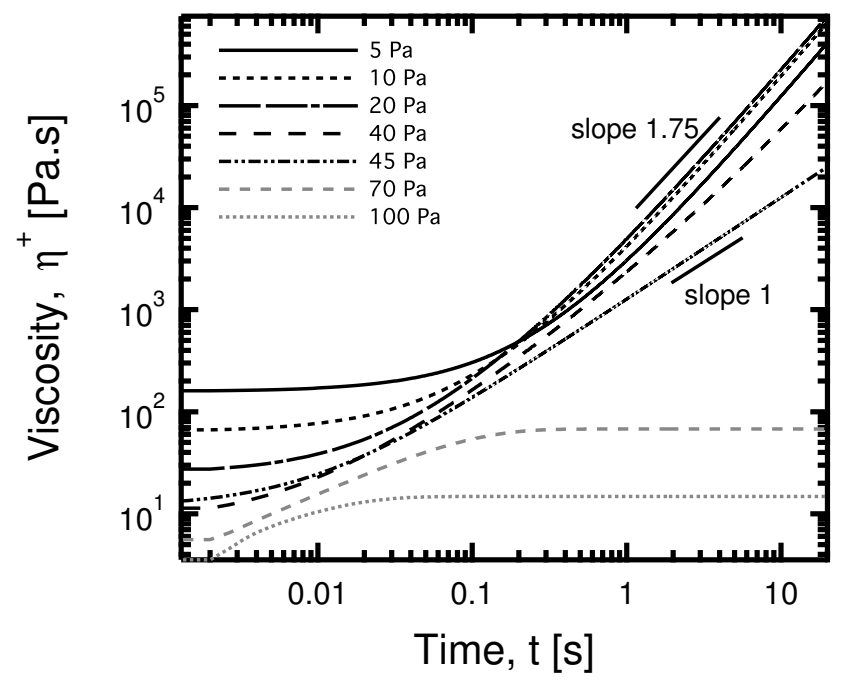

FIG. 14. Plot showing evolution of the apparent viscosity $\eta^{+} \equiv \sigma_{0} / \dot{\gamma}(t)$ for the KH model at a number of different imposed stresses above and below the value $\sigma_{y}=C / q=45 \mathrm{~Pa}$. Model parameters for the KH model were $C=540 \mathrm{~Pa}, q=12, k=26 \mathrm{~Pa} . \mathrm{s}^{m}, G=350 \mathrm{~Pa}, m=0.43$.

In Fig. 14 we show that the KH model predicts creep behavior that is qualitatively similar to that exhibited by the Carbopol microgel shown in Fig. 6 (b). For imposed stresses larger than the critical value $\sigma_{y}=C / q$, the viscosity approaches a steady state rather quickly, and this steady state dependency of viscosity on stress is governed by the Herschel-Bulkley equation given in Eq. 18. For values of the stress $\sigma<C / q$, continued power-law growth of the instantaneous viscosity is seen with time. The power-law exponent for this particular set of fitting parameters is determined to be to 1.75. An asymptotic expansion of Eqns. 25-28 under constant applied stresses $\sigma<\sigma_{y}$ yields the following scaling:

$$
\eta^{+} \simeq \eta_{c}\left(\frac{t}{t_{c}}\right)^{1 /(1-m)}
$$

This scaling holds for values of the imposed stress below the yield stress $C / q$, for values 
of $0<m<1$ and for long times. When the imposed stress $\sigma$ is exactly equal to the yield stress i.e. $\sigma=\sigma_{y}=C / q$, the constitutive equations predict a linear growth in viscosity with time, with $\eta \sim C t / m$ (this linear dependency can be identified as the dividing line of slope 1 in Fig. 14). The parameters $t_{c}$ and $\eta_{c}$ in Eq. 29 represent, respectively, a characteristic time scale and a characteristic viscosity scale for the KH model. They can be determined through the asymptotic expansion to be:

$$
\begin{aligned}
t_{c} & =\left(\frac{k}{C}\right)^{1 / m} \\
\eta_{c} & =\sigma\left(\frac{k}{C}\right)^{1 / m}\left[\left(1-\frac{q \sigma}{C}\right)\left(\frac{1-m}{m}\right)\right]^{1 /(1-m)}
\end{aligned}
$$

This particular model is therefore capable of capturing the power-law like growth in the instantaneous viscosity that we have observed in Carbopol. The slope of 1.75 in Fig. 14 is somewhat larger than the slope seen for Carbopol in Fig. 6 (b). The parameter $m$ can be modified in order to model the correct rate of increase of $\eta^{+}$with time, however for the purposes of fitting the model to LAOS data in the upcoming figures, we found a better agreement when we used the value of $m$ determined from the Herschel-Bulkley fit in Fig. 5 (a). This is reasonable because for the LAOS data (where strains are large) it is of primary importance to capture the behavior of the post-yielded flow regime, while small variations in the creeping behavior which occur below the yield stress do not play as important a role in the fitting of the constitutive model parameters.

One interesting consequence of this type of power-law growth or viscosity bifurcation is that it is possible for the model to capture a limiting viscosity plateau for $\sigma \leq \sigma_{y}$ in plots of apparent viscosity vs. imposed shear stress. These types of plateaus have been documented for Carbopol, and often spark debates as they beg the question as to whether or not a material truly exhibits a yield stress (Barnes, 1999; Møller et al., 2009). Fig. 15 shows a plot of viscosity vs. shear stress obtained from creep simulations carried out on the kinematic hardening model (with the same fitting parameters as Fig. 14) at a number of different imposed stresses. For each stress, the simulation is stopped after a given period of time, and the viscosity is instantaneously sampled. Viscosity plateaus are plotted for a number of different values of the dimensionless time $\hat{t} \equiv t / t_{c}$. From Fig. 15 it is clear that the material exhibits a time dependent plateau in the shear viscosity at stresses $\sigma<C / q$. 
The magnitude of this viscosity plateau increases as the measurement time is increased and diverges as $\hat{t} \rightarrow \infty$. The viscosity measurements at stresses $\sigma>C / q$ however, are insensitive to measurement time and converge to the Herschel-Bulkley equation quickly. Additionally it can be noted that the numerical value of apparent viscosity varies non-monotonically with the applied stress $\sigma$ at a fixed value of $\hat{t}$. This is due to the non monotonic variation of $\eta_{c}$ with $\sigma$ which is given in Eq. 31.

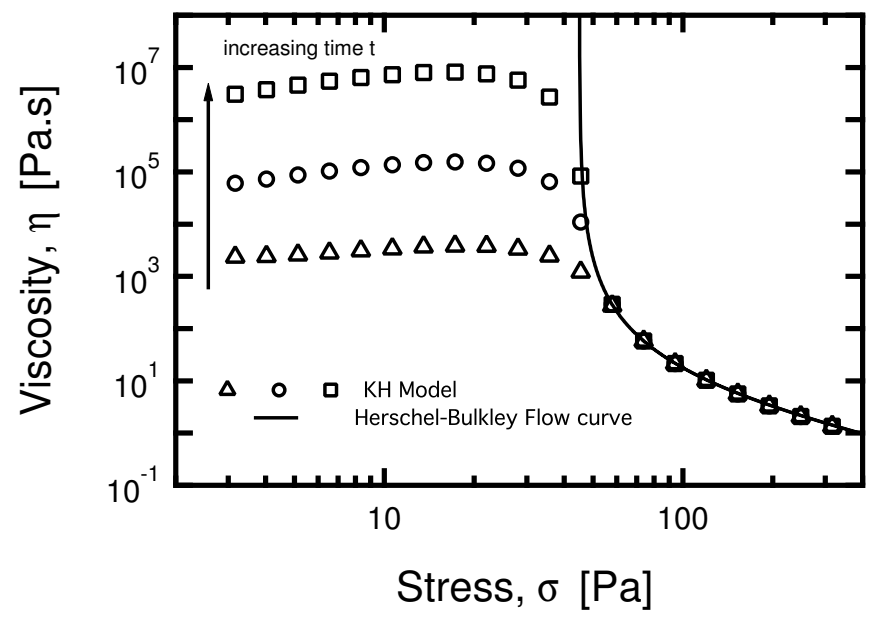

FIG. 15. Plot showing apparent flowcurve of the viscosity predicted by the KH model after different wait times. Flowcurves are obtained through simulations at a constant imposed stress, the viscosity is sampled at dimensionless times of $\hat{t}=1.2 \times 10^{3}(\triangle), 1.2 \times 10^{4}(\bigcirc)$, and $1.2 \times 10^{5}(\square)$ after the stress is imposed (corresponding to $t=1,10$ and 100 seconds). The overlaid solid line is the Herschel-Bulkley flow curve with the same values of model parameters $m, G$ and $k$ as the $\mathrm{KH}$ model, and with $\sigma_{y}=C / q=45 \mathrm{~Pa}$.

We have thus shown that the kinematic hardening model is capable of capturing several aspects of the rheological behavior of an ideal yield stress fluid such as Carbopol. It is considerably more versatile and realistic than the simpler EHB model, and only introduces one additional material constant. For the KH model, the plastic strain rate $\dot{\gamma}^{p}$ always varies continuously with the stress, and there are no conditionals on the evolution equations (such as the one in Eq. 19) to determine whether or not plastic flow will occur. Furthermore, as a result of the differential equation that defines the evolution in the parameter $A$, we recover a "viscosity bifurcation" (Coussot et al., 2002) with power-law-like growth in viscosity below a critical stress $C / q$, and convergence of the viscosity to a steady state value given by Eq. 18 above the critical stress $C / q$. It is therefore possible for the material to evolve towards two different states, with these states being separated by a "yield stress" determined by the ratio 
of the parameters $C / q$.

\section{E. KH Model under LAOStress}

So far, we have only studied the behavior of the kinematic hardening model under constant applied stress conditions. It is also possible to test how well the model fares at capturing flow behavior of the Carbopol gel under dynamic conditions, i.e. stress-controlled LAOS. In Fig. 16, we have overlaid the response of the kinematic hardening model (black) onto the response of the Carbopol (orange) on a Pipkin diagram.

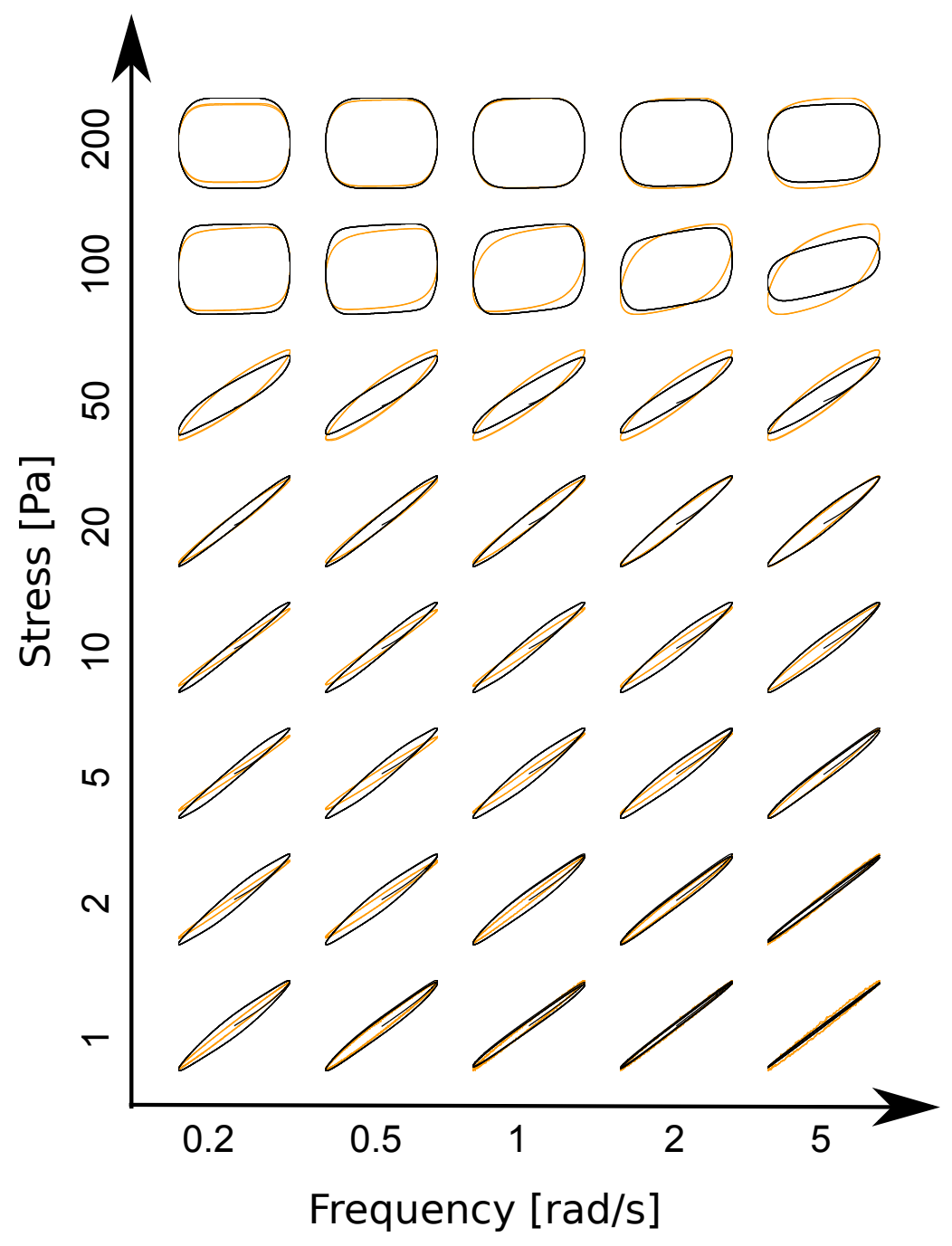

FIG. 16. Pipkin diagram showing comparison of kinematic hardening model (black) with Carbopol data (orange) at a number of different frequencies $\omega$ and stress amplitudes $\sigma_{0}$. The KH model fitting parameters are the same as those used in Figs. $12-15$ and are the same as the EHB fitting parameters in Fig. 11 with $\sigma_{y}=C / q$. 
It is clear from Fig. 16 that the kinematic hardening model performs considerably better than the EHB model in capturing the material response to LAOStress conditions. Both models predict approximately the same behavior at the largest stress amplitude, $\sigma_{0}=200$ $\mathrm{Pa}$, and the agreement between both models and the Carbopol data is excellent for this value of $\sigma_{0} \gg \sigma_{y}$. This is primarily a testament to the effectiveness of the Herschel-Bulkley model in capturing the steady state flow viscosity. However, the kinematic hardening model offers a considerable improvement over the EHB model in terms of modeling the measured behavior at moderate stress values of $\sigma \leq 50 \mathrm{~Pa}$. This improvement is due to the ability of the $\mathrm{KH}$ model to admit plastic flow for imposed stresses below the value $C / q$, which the EHB model does not account for. It is also important to emphasize the fact that the values of the fitting parameters $m, k$ and the ratio $C / q$ are constrained by the fit of the steady shear data in Fig. 5 to the Herschel-Bulkley model. Thus, the kinematic hardening model has effectively linked the transient nonlinear behavior under LAOS to steady state flow measurements such as those in Fig. 5.

By inspecting Fig. 16 and comparing it to Fig. 11 it is also clear that the variations in the material measures $J_{M}^{\prime}$ and $J_{L}^{\prime}$ at all of the tested frequencies are more accurately represented by the kinematic hardening model. The evolution in the values of $J_{M}^{\prime}$ and $J_{L}^{\prime}$ for the kinematic hardening model (plotted in Fig. 12) are related to the variation in the elastic and plastic strains defined in the constitutive model, $\gamma^{e}$ and $\gamma^{p}$. In particular, as a result of the differential equation relating $\dot{\gamma}^{p}$ to the defect parameter $A, \dot{\gamma}^{p}$ is no longer a single-valued function of the stress $\sigma$. It is important to note that the apparent plastic strain $\gamma^{\prime \prime}$ given by the strain decomposition in Eq. 9 is therefore different from $\gamma^{p}$, because the strain rate $\dot{\gamma}^{\prime \prime}$ given by Eq. 9 must be a single-valued function of $\sigma$ by construction (see Appendix). Monitoring and quantifying changes in the values of nonlinear measures such as $J_{M}^{\prime}$ and $J_{L}^{\prime}$ in LAOStress can help in understanding the evolution in internal parameters (like $A$ or the back stress $\sigma_{\text {back }}$ in the $\mathrm{KH}$ model) and this provides insight into how one might approach more sophisticated constitutive modeling of these kinds of soft materials.

To illustrate this, in Fig. 17 we present contour plots of the nonlinear material measures $J_{M}^{\prime}\left(\omega, \sigma_{0}\right)$ and $J_{L}^{\prime}\left(\omega, \sigma_{0}\right)$ for the kinematic hardening model. These can be contrasted with the contour plots of the measured values of $J_{M}^{\prime}$ and $J_{L}^{\prime}$ for Carbopol in Fig. 9. The general qualitative behavior of the model and the microgel is similar. For example, for the minimum stress compliance $J_{M}^{\prime}$, both model and data exhibit an initial increase and then a subsequent 
decrease as the stress amplitude $\sigma_{0}$ is increased. However, there is a stronger frequency dependence predicted for the kinematic hardening model - this can be discerned from the slopes of the isocontours. For the large stress compliance $J_{L}^{\prime}$, both model and data show an initial increase to a value larger than that of $J_{M}^{\prime}$, i.e. the material softens (or becomes more compliant) at large stresses. Again, the kinematic hardening model shows a stronger frequency dependence than the measurements, and also the maximum value of $J_{L}^{\prime}$ predicted by the kinematic hardening model is smaller than that observed experimentally.
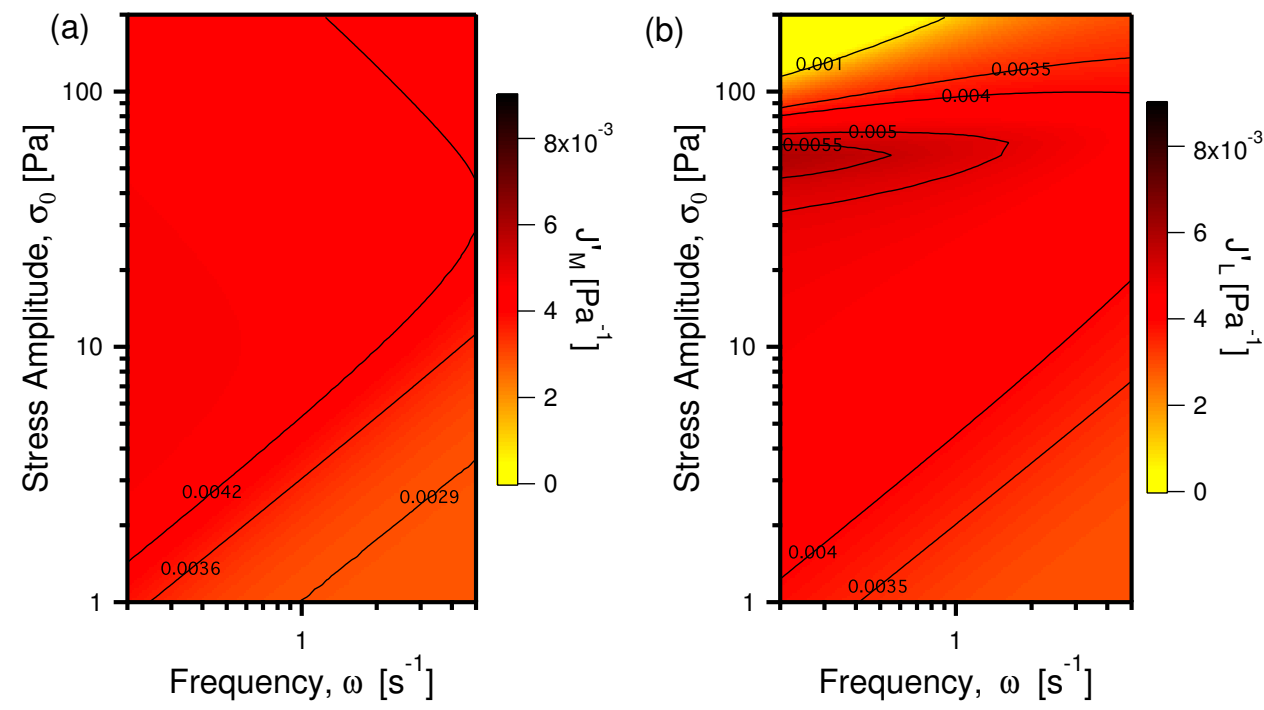

FIG. 17. Contour plots of the nonlinear material measures $J_{M}^{\prime}$ and $J_{L}^{\prime}$ for the KH model with the same fitting parameters used in Fig. 16. Qualitatively similar features to the contour plots of the Carbopol microgel in Fig. 9 can be seen in these contour plots.

For completeness, we add contour plots of the fluidities $\phi_{L}^{\prime}$ and $\phi_{M}^{\prime}$ for this model so that they can be contrasted with the experimental data. The simpler EHB model was already able to capture the stress-dependent variation of the large strain measure $\phi_{L}^{\prime}$ (which is dominated by viscoplastic flow), so the kinematic hardening model achieves an equally good agreement as the EHB model does with the Carbopol data. The improvement over the EHB model comes primarily from the non-zero values of $\phi_{M}^{\prime}$ when kinematic hardening is added to the constitutive model. Progressively larger values of $\phi_{M}^{\prime}$ at large values of $\sigma_{0}$ and $\omega$ are exhibited by the kinematic hardening model. This variation of $\phi_{M}^{\prime}$ is an important aspect of the material response in LAOS. Physically, it can be related to the time-varying nature of the Carbopol gel and its ability to exhibit viscoplastic flow, even at low stresses. The kinematic hardening model captures this behavior through the dynamic evolution of 

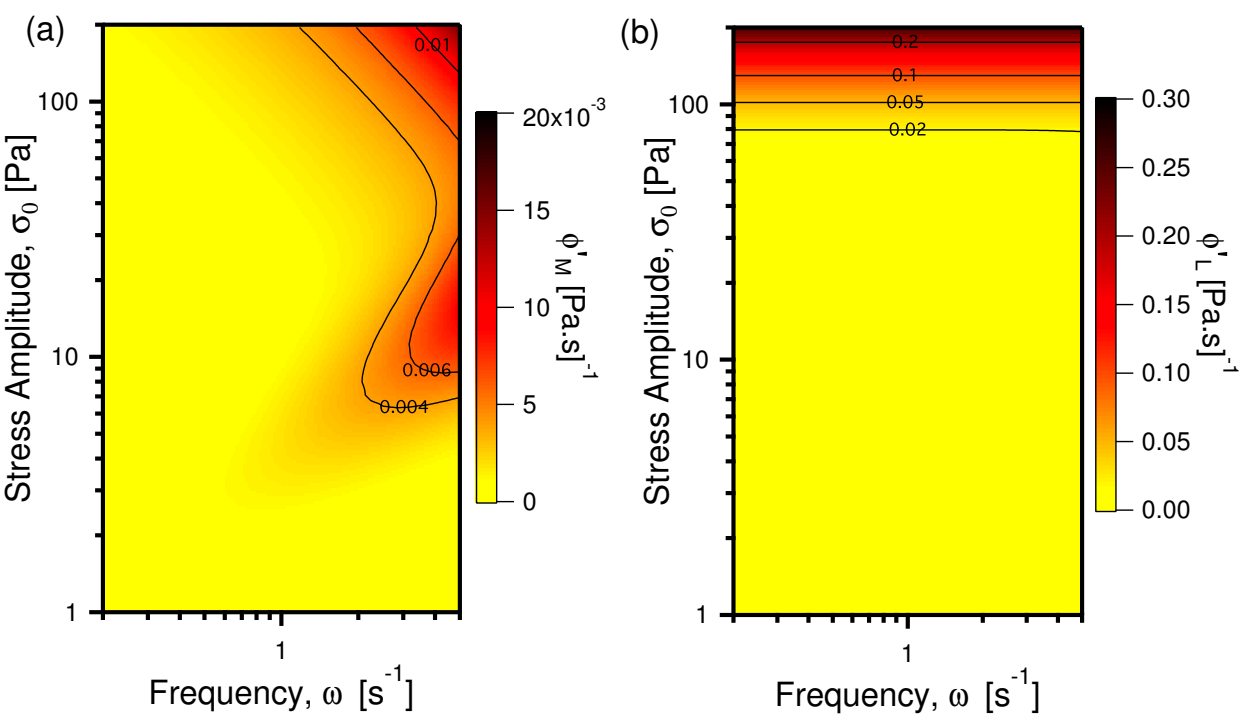

FIG. 18. Contour plots of the nonlinear measures $\phi_{M}^{\prime}$ and $\phi_{L}^{\prime}$ for the $\mathrm{KH}$ model with the same fitting parameters as in Fig. 16.

the back stress $\sigma_{\text {back }}(t)$ with characteristic time scale $t_{c}$ (Eq. 31), and the modified flow rule in Eq. 27. These features of the KH model allow the material to exhibit nonzero fluidity at low instantaneous stresses, even though under "steady state" conditions (i.e. creep tests in which we wait extremely long periods of time $t \gg t_{c}$ to measure an apparent viscosity) we would have $\eta^{+} \rightarrow \infty$ and fluidity $\phi^{+} \rightarrow 0$. The simpler EHB model attains this steady state instantaneously, and therefore predicts a constant (zero) value of $\phi_{M}^{\prime}$.

A definitive way to document the improved fit of the $\mathrm{KH}$ model vs. to the experimental data is by calculating the volumetric energy dissipated into the material per cycle, $E_{d}$. This energy can be computed by evaluating the following integral:

$$
E_{d}=\int_{0}^{2 \pi / \omega} \sigma \dot{\gamma} d t=\sigma_{0}^{2} J_{1}^{\prime \prime} \pi
$$

where the second equality follows due to the orthogonality of the Fourier terms in Eq. 7 over the domain $[0,2 \pi / \omega]$. It is well known that, for a sinusoidal forcing, the energy dissipated within the material per cycle only depends on the first viscous Fourier coefficient (Ganeriwala and Rotz, 1987; Kalelkar et al., 2010; Ewoldt et al., 2010). Geometrically, this parameter $E_{d}$ represents the area enclosed within the elastic Lissajous curves shown in Fig. 16. This measure provides the starkest contrast between the EHB and KH model fits to the Carbopol data, because for $\sigma<\sigma_{y}$ the EHB model does not dissipate energy at all - it behaves as 
an ideal elastic solid. In Fig. 19 we evaluate contour plots of $E_{d}\left(\omega, \sigma_{0}\right)$ and see that indeed, the increase in $E_{d}$ near $\sigma_{y}$ for the EHB model is very abrupt. On the other hand, $E_{d}$ increases more gradually for both the kinematic hardening model and the microgel data. To further emphasize this, in Fig. 19 (d) we compare experimental values of $E_{d}$ for the Carbopol microgel to predictions of the KH and EHB model with the same fitting coefficients used in Fig. 16 at a constant frequency of $\omega=5 \mathrm{rad} / \mathrm{s}$. While the EHB model fails to predict the energy dissipation for stress amplitudes below $80 \mathrm{~Pa}$, the kinematic hardening model shows excellent agreement with the measured values of $E_{d}$ for the Carbopol even for stresses $\sigma_{0} \ll \sigma_{y}$.

(a)

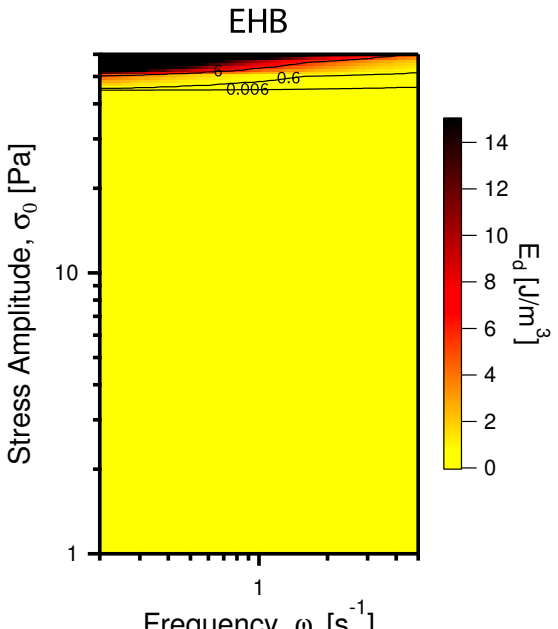

(c)

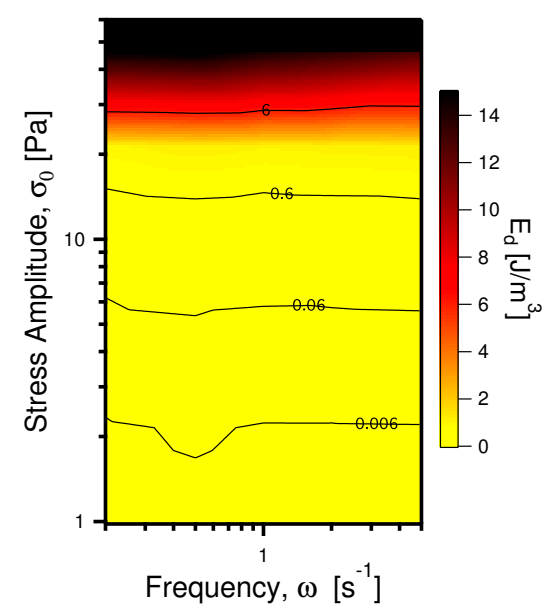

(b)

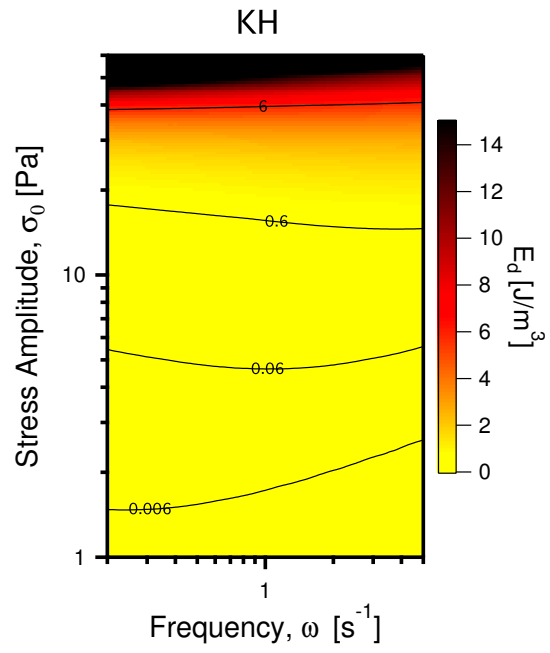

(d)

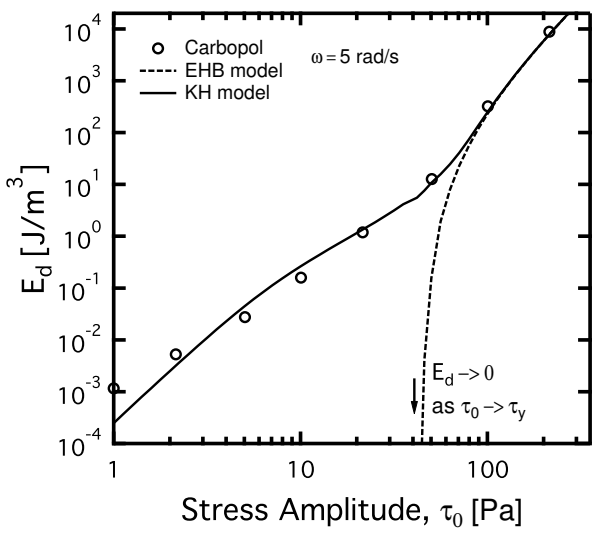

FIG. 19. Contour plot of the energy dissipated per cycle $\left(E_{d}\right)$ in (a), the EHB model plotted in Fig. 11, in (b), the kinematic hardening model plotted in Fig. 16 and in (c), the Carbopol microgel. In (d), a comparison of the predictions of both models is given with LAOStress data for the Carbopol microgel at an imposed frequency of $\omega=5 \mathrm{rad} / \mathrm{s}$. 


\section{CONCLUSIONS}

The goal of this work has been to show how stress-controlled LAOS (or LAOStress) can be used to extract meaningful nonlinear measures that quantify the response of elasto-plastic materials to oscillatory deformations. In general these descriptive rheological measures are not identical to prescriptive constitutive model parameters, but they are related. A further goal has therefore been to show how these material measures can be used to develop and assess quantitative constitutive models that can predict yield-like responses of gels and other soft solids. To that end, we have introduced the stress-controlled LAOS framework in Sec. III. This framework is identical to that first used by Ewoldt (2009) and Läuger and Stettin (2010), and has been extended to include the Chebyshev coefficients $c_{n}$ and $f_{n}$ which characterize the nonlinear compliance and fluidity of a material.

We have discussed the implications of a stress vs. strain decomposition of the material response, and how it may be physically more meaningful for gels and soft solids to adopt the stress-controlled approach in which strain is decomposed into elastic and plastic components. This strain decomposition into "apparent" components $\gamma^{\prime}$ and $\gamma^{\prime \prime}$ is intimately related to the concepts of elastic and plastic strains defined for certain classes of constitutive models. However, with more complex constitutive models, the specific relation between these apparent strains and the corresponding parameters $\left(\gamma^{e}\right.$ and $\left.\gamma^{p}\right)$ in a model is not straightforward (i.e they may not be equal to each other). The Chebyshev compliance and fluidity coefficients have been defined in this work, but we have refrained from using them to quantify material behavior for two reasons. The first reason is due to the potential differences between the experimentally-decomposed strains (i.e. the descriptive measures $\gamma^{\prime}$ and $\gamma^{\prime \prime}$ ) and the constitutive model strain decomposition (prescriptive measures $\gamma^{e}$ and $\gamma^{p}$ ), and the implications that this has for prescribing a particular constitutive behavior. Second, we are generally interested in very large nonlinearities in the rheological fingerprint (e.g. yielding) where it has been shown (Klein et al., 2007; Ewoldt et al., 2010; Rogers et al., 2011b) that harmonics higher than $n=3$ can play a significant role in the material response. As a result we have focused our efforts on measuring and predicting the nonlinear compliances $J_{M}^{\prime}$ and $J_{L}^{\prime}$, and the nonlinear fluidities $\phi_{M}^{\prime}$ and $\phi_{L}^{\prime}$ which involve all the Fourier-Chebyshev coefficients. Expressions exist for each of these nonlinear measures in terms of the higher order Fourier compliances $J_{n}^{\prime}$ and $J_{n}^{\prime \prime}$ - see Eqs. 12, 14, 16, 17). We have shown that the nonlinear com- 
pliance $J_{M}^{\prime}$ serves as a better indicator of the intracycle elasticity of the material below the yield stress than the equivalent strain-controlled measure, $G_{M}^{\prime}$, which decreases by orders of magnitude after the material yields. A new interpretation of $G_{M}^{\prime}$ is therefore suggested, i.e. it measures the residual material elasticity during flow at the maximum strain rate. The parameters $J_{M}^{\prime}$ and $G_{M}^{\prime}$ are thus complements to each other but not conjugates. For stress-controlled LAOS conditions, the value of $J_{M}^{\prime}$ remains relatively stable for all stress amplitudes and frequencies. This behavior is consistent with that of an elasto-plastic material, in which strain is decomposed into elastic and plastic components, and elastic deformations occur "in series" with viscoplastic deformations at all stress amplitudes. For Carbopol, the nonlinear fluidity $\phi_{L}^{\prime}$ increases dramatically after yield due to the viscoplastic flow in the material which occurs above the yield stress.

Based on these observations, we have introduced two constitutive models that can be used to capture the behavior of the Carbopol microgel. The first of these models, the ElasticHerschel-Bulkley model (EHB), is successful at capturing the behavior of the material under LAOS conditions at very large stress amplitudes and at very small stress amplitudes. However, this model was shown to be deficient from the perspective of the nonlinear measures $J_{M}^{\prime}, J_{L}^{\prime}$ and $\phi_{M}^{\prime}$ - specifically, it predicts constant values for all of these measures. To improve on this shortcoming, we modified the EHB model in order to account for kinematic hardening. This constitutive process involves the introduction of an evolving internal material parameter which is defined through a nonlinear differential equation. This internal parameter, known as the back stress, corresponds to the location of the center of the yield surface of the material in stress space. The dynamics introduced through this equation allow the material to exhibit a continuous transition under LAOS from the unyielded to the yielded regime. While the simpler EHB model exhibits a sudden change in behavior for stresses in the vicinity of the yield stress $\sigma_{y}$, the ability of the $\mathrm{KH}$ model to evolve over time in a continuous manner prevents this discontinuous change in behavior from occurring. The KH model is able to capture many of the salient features of the Carbopol response - in particular, below the yield stress it predicts a power-law growth in the instantaneous viscosity with time, a behavior which has been documented experimentally by several groups. Above the yield stress, the model predicts a steady state flowcurve given by the Herschel-Bulkley equation. The corresponding yield stress at which this viscosity bifurcation occurs is easily tunable in the model by altering the ratio of the material constants $C / q=\sigma_{y}$. The kinematic hard- 
ening model also predicts nonzero viscous dissipation within the material at stresses below the yield stress. This is due to the ability of the model to exhibit slow viscoplastic creep at low stresses $\sigma<\sigma_{y}$.

Looking beyond the present work, there are several interesting new avenues for research that can be pursued. One potentially interesting area of work would be modifying the kinematic hardening model to account for the more complex thixotropic behavior (Barnes, 1997) that is exhibited by some of the less "ideal" yield stress fluids (such as the bentonite suspensions studied by Coussot et al. (2002)). There have been many examples of recent work in which models have been proposed to account for thixotropy (Yziquel et al., 1999;

Mujumdar et al., 2002; Alexandrou et al., 2009; de Souza Mendes, 2011), and it would be of interest to see how thixotropic behavior can be implemented into the kinematic hardening model. These types of fluids also present new experimental challenges related to LAOS implementation - de-structuring of thixotropic fluids may lead to long transients in the LAOS signal which do not die out until multiple periods of oscillation have been completed. These transients are readily visible in the Lissajous curves, but a quantitative description will require more advances in the LAOS framework, including the use of even harmonics to fully capture the transient responses.

Finally, it would be interesting to compare and draw analogies between some of the microstructure modeling efforts that have been carried out on soft materials (e.g. Coussot (2007)), to analogous modeling of materials that are more commonly dealt with in the plasticity literature, such as polycrystalline metals (see Rubin and Yarin (1993) for an introductory discussion). In the present paper we have seen that the kinematic hardening model employed in plastic deformation of metals is successful in predicting the nonlinear response of the Carbopol microgel under cyclic loading. Thus, further comparisons with inspiration drawn from the plasticity literature may prove insightful for rheological descriptions of soft solids, gels and other yield stress fluids.

\section{ACKNOWLEDGMENTS}

The authors would like to thank L. Anand for his insightful assistance with the modeling of the nonlinear response of the microgel. In addition to this, we would also like to thank Dr. Will Hartt and Lori Bacca of Procter and Gamble for providing the Carbopol sample. We 
would also like to thank Aly Frank at TA instruments for bringing some of the inertia related issues associated with stress-controlled LAOS measurements on the ARG2 to our attention. CJD would also like to thank Chevron Energy Technology Corporation for providing funding.

\section{Appendix A: Generalization of Kinematic Hardening Model to 3D Finite Deformations}

Both the EHB model, and the kinematic hardening model can be generalized to 3dimensional form, such that the constitutive models are frame invariant and satisfy the first and second laws of thermodynamics. The nomenclature used and the approach taken here is very similar to that taken by Gurtin et al. (2010), so readers who are interested in additional details are advised to consult those works. Lemaitre and Chaboche (1990) is also a good general reference for readers interested in various types of kinematic hardening models. For the sake of brevity, this appendix will mostly serve to highlight some of the important concepts of the model; Henann and Anand (2009), Anand et al. (2008) and Ames et al. (2009) discuss more complex versions of the model that is detailed in this appendix. Specifically, the model discussed here does not include isotropic hardening, and is not thermo-mechanically coupled as is the case for more general models.

A central component of this generalization is the Kroner decomposition (Kroner, 1960), in which the deformation gradient, $\mathbf{F}$, is multiplicatively decomposed into elastic and plastic components, $\mathbf{F}^{e}$ and $\mathbf{F}^{p}$ respectively.

$$
\mathbf{F}=\mathbf{F}^{e} \mathbf{F}^{p}
$$

This plays the same role that the additive decomposition of Eq. 1 plays for the 1-D case. The polar decomposition of the elastic component of the deformation gradient is as follows:

$$
\mathbf{F}^{e}=\mathbf{R}^{e} \mathbf{U}^{e}
$$

With $\mathbf{R}^{e}$ representing a rotation and $\mathbf{U}^{e}$ a stretch. The stretch $\mathbf{U}^{e}$ has the following spectral representation:

$$
\mathbf{U}^{e}=\sum_{i=1}^{3} \lambda_{i}^{e} \mathbf{r}_{i}^{e} \otimes \mathbf{r}_{i}^{e}
$$


Where $\lambda_{i}^{e}$ are the principal values and $\mathbf{r}_{i}^{e}$ are the principal directions of $\mathbf{U}^{e}$. From the stretch $\mathbf{U}^{e}$ we can therefore define the logarithmic elastic strain (Hencky strain) which is as follows:

$$
\mathbf{E}^{e} \equiv \ln \mathbf{U}^{e}=\sum_{i=1}^{3}\left(\ln \lambda_{i}^{e}\right) \mathbf{r}_{i}^{e} \otimes \mathbf{r}_{i}^{e}
$$

The use of the logarithmic elastic strain is typically done in order to approximately capture elastic behavior at large strains. In addition to the definition of $\mathbf{E}^{e}$, we can define the right $\left(\mathbf{C}^{e}\right)$ and left $\left(\mathbf{B}^{e}\right)$ elastic Cauchy-Green tensors as follows:

$$
\begin{aligned}
& \mathbf{C}^{e}=\left(\mathbf{F}^{e}\right)^{\top} \mathbf{F}^{e} \\
& \mathbf{B}^{e}=\mathbf{F}^{e}\left(\mathbf{F}^{e}\right)^{\top}
\end{aligned}
$$

The left Cauchy-Green tensor is also sometimes referred to as the Finger tensor (Bird et al., 1987; Macosko, 1994).

\section{Elastic Herschel-Bulkley Model}

For the simpler EHB model, elastic strains are assumed to be small, so the free energy can be written as a function of $\mathbf{E}^{e}$, and the following specific form is assumed:

$$
\Psi=G\left|\mathbf{E}^{e}\right|^{2}+\frac{1}{2} \lambda\left|\operatorname{tr} \mathbf{E}^{e}\right|^{2}
$$

Eq. A7 above has introduced the parameter $\lambda$ as an additional material parameter which was not needed for the 1 dimensional case of simple shear discussed in Sec. IV. It is related to the bulk modulus $K$ through $K=\lambda+2 G / 3$. Eq. A7 results in the following form for the second Piola elastic stress $\mathbf{T}^{e}$ :

$$
\mathbf{T}^{e}=2 G \mathbf{E}^{e}+\lambda\left(\operatorname{tr} \mathbf{E}^{e}\right) \mathbf{1}
$$

Where $\mathbf{T}^{e}$ is defined as follows:

$$
\mathbf{T}^{e}=J\left(\mathbf{F}^{e}\right)^{-1} \mathbf{T}\left(\mathbf{F}^{e}\right)^{-\top}
$$


Where $\mathbf{T}$ is the Cauchy stress and $J=\operatorname{det}(\mathbf{F})$.

The next step is to formulate a plastic flow law which determines the rate of plastic flow given an applied stress. For the EHB model we will use a rate dependent plastic flow rule. We begin with the plastic velocity gradient $\mathbf{L}^{p}$ which is related to $\mathbf{F}^{p}$ as follows:

$$
\mathbf{L}^{p}=\dot{\mathbf{F}}^{p}\left(\mathbf{F}^{p}\right)^{-1}
$$

$\mathbf{L}^{p}$ can be split into its symmetric and skew components, such that $\mathbf{L}^{p}=\mathbf{D}^{p}+\mathbf{W}^{p}$. One of the assumptions that goes into this model is that of plastic irrotationality, which assumes $\mathbf{W}^{p}=0$, i.e. there is no plastic spin. We can then write the plastic stretching $\mathbf{D}^{p}$ as a product of its magnitude, $d^{p}=\left|\mathbf{D}^{p}\right|$, and its direction $\mathbf{N}^{p}=\mathbf{D}^{p} / d^{p}$.

$$
\mathbf{D}^{p}=d^{p} \mathbf{N}^{p}
$$

Note that $\mathbf{N}^{p}$ is the 3D tensor-valued analog of the direction $n^{p}$ discussed in the $1 \mathrm{D}$ version of the model (see Eq. 19). In order to determine $\mathbf{N}^{p}$, we assume that plastic flow is codirectional with the stress. However, this codirectionality holds for a different projection of the total stress, the deviatoric Mandel stress $\mathbf{M}_{0}^{e}$ (Gurtin et al., 2010). Thus:

$$
\mathbf{N}^{p}=\frac{\mathbf{M}_{0}^{e}}{\left|\mathbf{M}_{0}^{e}\right|}
$$

Where the Mandel stress $\mathbf{M}^{e}$ is associated with an intermediate "structural" space in the material (Gurtin et al., 2010) and is defined as:

$$
\mathbf{M}^{e}=\mathbf{C}^{e} \mathbf{T}^{e}
$$

And its deviatoric part is $\mathbf{M}_{0}^{e}=\mathbf{M}^{e}-\frac{1}{3}\left(\operatorname{tr} \mathbf{M}^{e}\right) \mathbf{1}$. We can then define an equivalent plastic strain rate and equivalent shear stress:

$$
\begin{array}{lr}
\dot{\bar{\gamma}}^{p}=\sqrt{2} d^{p} & \text { Equivalent plastic strain rate } \\
\bar{\sigma}=\frac{1}{\sqrt{2}}\left|\mathbf{M}_{0}^{e}\right| & \text { Equivalent shear stress }
\end{array}
$$

The presence of the $\sqrt{2}$ factor in Eq. A14 arises so that under simple shearing conditions, 
the equivalent plastic strain $\dot{\bar{\gamma}}^{p}$ is equal to the plastic strain rate $\dot{\gamma}^{p}$ discussed for the $1 \mathrm{D}$ shear case (note that conventional strain rate tensor is in rheology is typically defined as 2D). In Eq. A15, the $\sqrt{2}$ factor arises as a result of the von-Mises yield criterion - the factor preserves the value of the yield stress $\sigma_{y}$ when the $3 \mathrm{D}$ model is simulated under simple shearing conditions. The positive, scalar valued parameters $\bar{\sigma}$ and $\dot{\bar{\gamma}}^{p}$ defined above are related through the familiar Herschel-Bulkley flow equation:

$$
\dot{\bar{\gamma}}^{p}= \begin{cases}0 & \text { if } \bar{\sigma}<\sigma_{y} \\ \left(\frac{\bar{\sigma}-\sigma_{y}}{k}\right)^{1 / m} & \text { if } \bar{\sigma} \geq \sigma_{y}\end{cases}
$$

Due to the presence of the $\sqrt{2}$ factors in the equivalent plastic strain rates and equivalent shear stress, this Herschel-Bulkley flow equation reduces to the 1D equation with identical values of the yield stress $\sigma_{y}$ and the fitting parameters $k$ and $m$ discussed in Sec. IV when the material is specialized to simple shearing conditions.

\section{Implementing Kinematic Hardening}

In order to introduce kinematic hardening into the model, the free energy $\Psi$ defined in Eq. A7 is modified by adding an additional term, such that:

$$
\Psi=G\left|\mathbf{E}^{e}\right|^{2}+\frac{1}{2} \lambda\left|\operatorname{tr} \mathbf{E}^{e}\right|^{2}+\underbrace{\Psi^{p}(\mathbf{A})}_{\text {defect energy }}
$$

The form of the free energy equation above involves the introduction of a defect energy, $\Psi^{p}$, which depends on the tensor $\mathbf{A}$ (which is the three-dimensional generalization of the parameter $A$ that was discussed in Sec. IVD). $\mathbf{A}$ is symmetric and unimodular (i.e. $\operatorname{det}(\mathbf{A})=1$, and has the following spectral representation:

$$
\mathbf{A}=\sum_{i=1}^{3} a_{i} \mathbf{l}_{i} \otimes \mathbf{l}_{i}
$$

Where the $\mathbf{l}_{i}$ are the principal directions of $A$. The following simple form of the $\Psi^{p}$ is used to model the defect energy:

$$
\Psi^{p}=\frac{1}{4} C\left[\left(\ln a_{1}\right)^{2}+\left(\ln a_{2}\right)^{2}+\left(\ln a_{3}\right)^{2}\right]
$$


In Eq. A19 the back stress modulus $C$ has been introduced, which is a new material parameter. Eqns A17 and A19 above result in the following equation for the back stress, $\mathrm{M}_{\mathrm{back}}^{e}$ :

$$
\mathbf{M}_{\mathrm{back}}^{e}=C \ln \mathbf{A}
$$

The parameter $\mathbf{A}$ is then defined through the following evolution equation:

$$
\dot{\mathbf{A}}=\mathbf{D}^{p} \mathbf{A}+\mathbf{A D}^{p}-q \mathbf{A}(\ln \mathbf{A}) d^{p}
$$

In this equation we have introduced the new material constant $q$, which is equivalent to the dimensionless $q$ parameter discussed for the 1D model in Sec. IV. The value of the parameter $q$ determines the dynamic recovery of $\mathbf{A}$. The effective stress driving the plastic flow, $\mathbf{M}_{\text {eff }}^{e}$, is then given by:

$$
\mathbf{M}_{\mathrm{eff}}^{e}=\mathbf{M}_{0}^{e}-\mathbf{M}_{\mathrm{back}}^{e}
$$

With kinematic hardening we assume that the plastic flow $\mathbf{D}^{p}$ is now codirectional with the effective stress $\mathbf{M}_{\text {eff }}^{e}$, so for the directional tensor $\mathbf{N}^{p}=\mathbf{D}^{p} / d^{p}$ we now have:

$$
\mathbf{N}^{p}=\frac{\mathbf{M}_{\text {eff }}^{e}}{\left|\mathbf{M}_{\text {eff }}^{e}\right|}
$$

The stress driving plastic flow in the EHB model must also be modified, to account for the backstress, so Eq. A15 becomes:

$$
\bar{\sigma}=\frac{1}{\sqrt{2}}\left|\mathbf{M}_{\text {eff }}^{e}\right|
$$

Finally, the power-law rate-dependent flow rule is introduced, which gives the relation between $\bar{\sigma}$ and $\dot{\bar{\gamma}}^{p}$ :

$$
\dot{\bar{\gamma}}^{p}=\left(\frac{\bar{\sigma}}{k}\right)^{1 / m}
$$

Note that this particular flow rule has eliminated the conditionality of Eq. A16, and thus eliminated the explicit appearance of the yield stress parameter $\sigma_{y}$. 


\section{Appendix B: Effect of Instrument Inertia on Stress-Controlled LAOS measurements}

When carrying out dynamic stress-controlled experiments on a single head rheometer such as an ARG2, the rheometer typically controls the total torque applied, which is distributed between the instrument geometry, and the sample. In the present work, the total torque (which was sampled before any filtering using an auxiliary sample program on the ARG2) was converted to the shear stress acting on the sample using an appropriate geometry factor ( $2 \pi R^{3} / 3$ with $R$ being the radius of the cone-plate geometry). For greater accuracy, one would want to subtract any contribution of the inertial torque from the measured signal before applying this geometry factor.

For small enough frequencies and strain amplitudes, we expect that influences from the inertial torque can be neglected and we seek to provide a dimensionless constrain that quantifies this expectation. The total torque $T$ can be additively decomposed into an 'inertia torque', $T_{i}$ and the sample torque, $T_{s}$ such that $T=T_{i}+T_{s}$. The inertia torque is related to the angular orientation of the geometry, $\theta$, and the instrument moment of inertia $I$ as follows:

$$
T_{i}=I \frac{d^{2} \theta}{d t^{2}}
$$

In order to be able to neglect the effects that instrument inertia has on an experiment, it is desired for the ratio $T_{i} / T_{s}$ to be small. For the particular experiments carried out in this work (stress-controlled oscillations with stress amplitude $\sigma_{0}$ and frequency $\omega$, in a cone and plate geometry), one can estimate the values of $T_{i}$ and $T_{s}$ as follows:

$$
\begin{aligned}
T_{s} & \sim \frac{2 \sigma_{0} \pi R^{3}}{3} \\
T_{i} & \sim I \omega^{2} \gamma_{0} \phi
\end{aligned}
$$

Where $R$ is the radius of the cone-plate geometry, $\phi$ is the cone angle, and $\gamma_{0}$ is the strain amplitude of the deformation, which naturally depends on the type of material being probed. We define a dimensionless inertia number In which estimates the relative magnitude of the inertia torque to the sample torque as follows: 


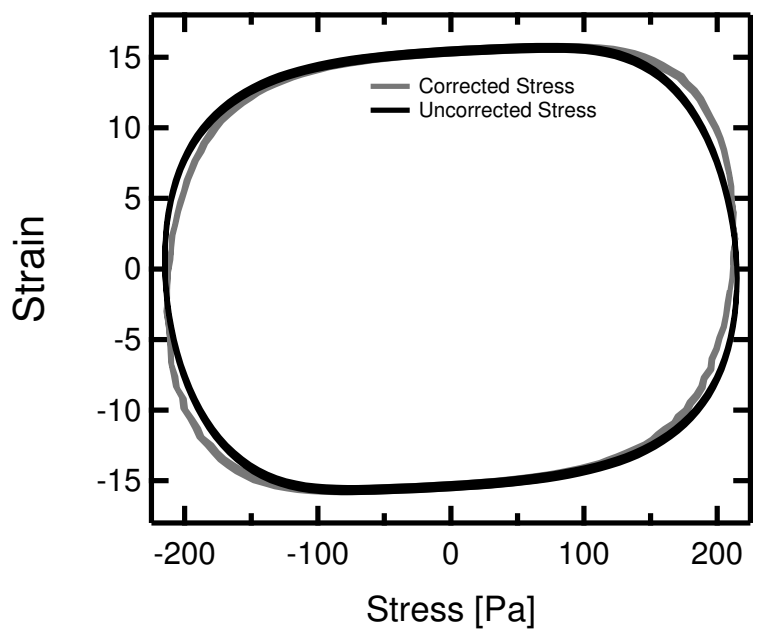

FIG. 20. Comparison of corrected and uncorrected Lissajous curves for LAOS experiment with $\omega=5 \mathrm{rad} / \mathrm{s}$ and $\sigma_{0}=200 \mathrm{~Pa}$

$$
I n=\frac{3 I \omega^{2} \phi \gamma_{0}}{2 \pi \sigma_{0} R^{3}}
$$

The strain amplitude of deformation cannot be determined a priori. For the present work we can determine $\gamma_{0}$ from the experiments presented in Fig. 8, and based on these values of $\gamma_{0}$, we find $I n<0.05$ for all the experiments. The test in Fig. 8 for which $I n$ is largest is that where the imposed frequency is $\omega=5 \mathrm{rad} / \mathrm{s}$ and the stress amplitude is $200 \mathrm{~Pa}$. In order to illustrate that the cutoff of $I n \leq 0.05$ is acceptable, we plot the corrected stress-strain curve on top of the uncorrected stress strain curve in Fig. 20. As Fig. 20 shows, there is little change in the overall shape of the Lissajous curve for this particular frequency. In particular, the value of $J_{M}^{\prime}$ evaluated from the two different curves differs by less than $3 \%$. However, a quantitative comparison of the measure $J_{L}^{\prime}$ at this particular value of $\omega$ and $\sigma_{0}$ is rather difficult to obtain, due to a substantial amount of noise being introduced into the corrected stress signal at higher values of $\sigma(t)$.

Another way to measure the importance of inertia in a stress-controlled test is by looking at the relative "purity" of the periodic torque wave $T_{s}(t)$ imposed on the sample. Note that for stress-controlled LAOS, the rheometer will typically impose a total torque such that $T(t)=T_{0} \cos \omega t$. Due to the nonlinear nature of the material response, the torsional angle $\theta(t)$ will contain higher harmonics. As a result, $T_{i}(t)$ will also contain higher harmonics, as a consequence of the definition in Eq. B1. There is therefore no guarantee that the oscillating 


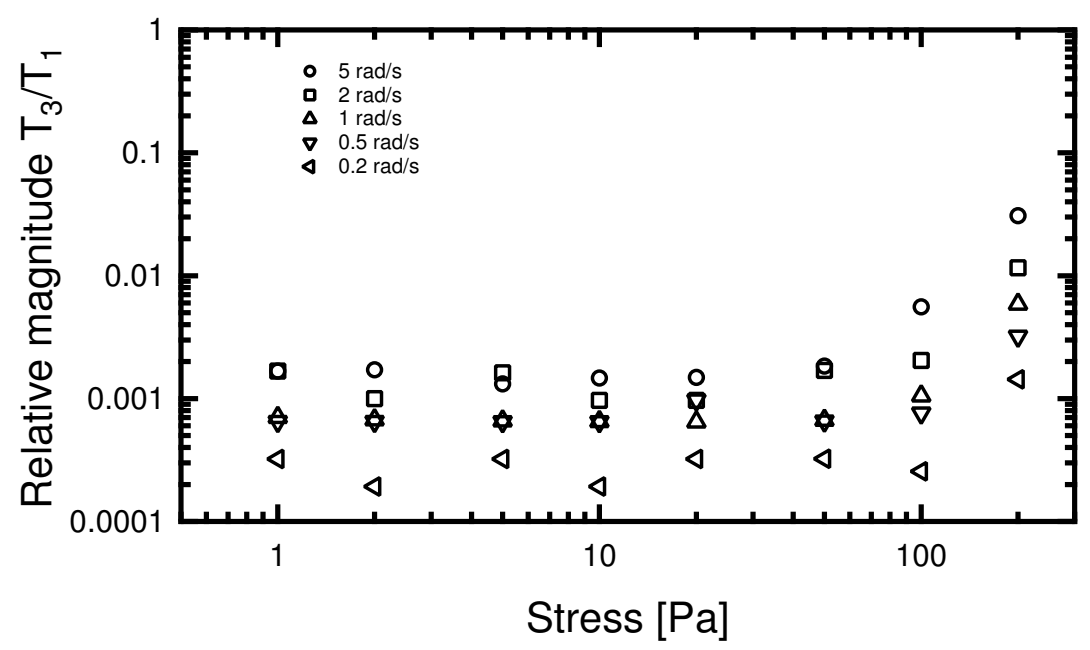

FIG. 21. Plot of the relative size of the third harmonic to the first harmonic in the periodic sample stress waveform, $T_{s}(t)$

torque, $T_{s}(t)=T(t)-T_{i}(t)$ applied to the sample will only contain a single harmonic in its waveform. For each of the experiments presented in this work, it is possible to correct $T(t)$ for inertia in order to obtain the true sample torque $T_{s}(t)$. An FFT of $T_{s}(t)$ can then be taken and the relative magnitude of the third harmonic to the first harmonic $\left(T_{3} / T_{1}\right)$ can be determined. This gives the LAOS experimentalist an idea of how pure the spectral content of the stress input into the material is. In Fig. 21 we show the ratio $T_{3} / T_{1}$ for the LAOStress measurements presented in this work. From the figure, it is apparent that the ratio increases beyond a noise threshold $(\sim 0.2 \%)$ for the highest stress amplitude, but never increases beyond $3 \%$ at the highest frequency and stress amplitude

It is also possible to make a coarse estimate of what the limiting strain amplitude, $\gamma_{0}$, would be so as to determine a value of this criterion before doing experiments. For values of $\sigma_{0}$ below the yield stress, we expect $\gamma_{0} \sim \sigma_{0} / G$ (i.e. the material will behave primarily as an elastic solid). For values of $\sigma_{0}$ above the yield stress, however, the material will deform with a maximum shear rate (given by the Herschel-Bulkley equation) of $\left(\frac{\sigma_{0}-\sigma_{y}}{k}\right)^{1 / m}$. If we multiply this shear rate by the period, $2 \pi / \omega$, we obtain an estimate for the total accumulated viscoplastic strain in the material. We also need to add the accumulated elastic strain to the material as well $\left(\sigma_{0} / G\right)$, so when we add these two terms we obtain the following estimate 
for $\gamma_{0}$ above the yield stress:

$$
\gamma_{0} \sim \frac{\sigma_{0}}{G}+\left(\frac{\sigma_{0}-\sigma_{y}}{k}\right)^{1 / m} \frac{2 \pi}{\omega}
$$

Using these estimates for the strain amplitude both below and above the yield stress, we can modify the dimensionless inertia number defined in Eq. B4. In the absence of any knowledge of $\gamma_{0}$, In can then be determined as follows for a soft solid gel:

$$
\begin{array}{ll}
\text { In }=\frac{3 I \omega^{2} \phi}{2 \pi R^{3} G} & \text { for } \sigma<\sigma_{y} \\
\text { In }=\frac{3 I \omega^{2} \phi}{2 \sigma_{0} \pi R^{3}}\left(\sigma_{0} / G+\left(\frac{\sigma_{0}-\sigma_{y}}{k}\right)^{1 / m} \frac{2 \pi}{\omega}\right) & \text { for } \sigma \geq \sigma_{y}
\end{array}
$$

A suitable upper bound constraint can then be placed on the value of $I n$, which allows the range of test frequencies and imposed stresses for which inertial effects are negligible to be evaluated before stress-controlled experiments are actually performed.

\section{Appendix C: Uniqueness and Single-Valued Properties of Strain Decomposition}

We consider the case in which we have a sinusoidally imposed stress $\sigma(t)$ as given in Eq. 6 (and inertial effects are negligible), with the strain $\gamma(t)$ being decomposed into a Fourier series as given in Eq. 7. Based on the Fourier decomposition of Eq. 7, we desire to find a strain decomposition $\gamma=\gamma^{\prime}+\gamma^{\prime \prime}$ such that $\gamma^{\prime}$ is a single-valued function of stress, and $\gamma^{\prime \prime}$ is a single-valued function of stress.

\section{Strain Decomposition}

We propose that the following decomposition satisfies the criteria of $\gamma^{\prime}$ being a singlevalued function of $\sigma$ and $\dot{\gamma}^{\prime \prime}$ being a single-valued function of $\sigma$ :

$$
\begin{aligned}
\gamma^{\prime}(t) & =\sum_{n \text { odd }} J_{n}^{\prime} \sigma_{0} \cos n \omega t \\
\gamma^{\prime \prime}(t) & =\sum_{n \text { odd }} J_{n}^{\prime \prime} \sigma_{0} \sin n \omega t
\end{aligned}
$$


In order to show this, we must show that if $\sigma\left(t_{1}\right)=\sigma\left(t_{2}\right)$, then $\gamma^{\prime}\left(t_{1}\right)=\gamma^{\prime}\left(t_{2}\right)$, i.e. for two different times at which the stress is equal, the value of $\gamma^{\prime}$ must be equal. For $\sigma\left(t_{1}\right)=\sigma\left(t_{2}\right)$ we must have $\cos \omega t_{1}=\cos \omega t_{2}$. Due to the fact that cosine is an even function, this only holds if:

$$
\omega t_{1}=-\omega t_{2}
$$

Using equation C3 we can obtain a relationship between the higher harmonics in equation C1 at times $t_{1}$ and $t_{2}$.

$$
\cos n \omega t_{1}=\cos \left(-n \omega t_{2}\right)=\cos n \omega t_{2}
$$

Where the last equality follows because cosine is an even function regardless of the value of $n$. As a result of equation $\mathrm{C} 4$, all the terms in equation $\mathrm{C} 1$ are the same if they are evaluated at either $t_{1}$ or $t_{2}$, as long as the stress is the same at times $t_{1}$ and $t_{2}$. Because of this, $\gamma^{\prime}$ is a single-valued function of stress. It follows that $\dot{\gamma}^{\prime \prime}$ is also a single-valued function of stress, because it consists of only cosine terms (it is the time derivative of sine terms), and the same logic applies.

\section{Uniqueness of Strain Decomposition}

We now show that the strain decomposition in equations $\mathrm{C} 1$ and $\mathrm{C} 2$ is unique, in the sense that no other strain decomposition gives an apparent elastic contribution $\gamma^{\prime}$ which is a single-valued function of the stress $\sigma$ and an apparent plastic strain rate $\dot{\gamma}^{\prime \prime}$ which is a single-valued function of the stress $\sigma$. In order to do this, we propose an alternative elastic strain, $\gamma^{1}$. This $\gamma^{1}$ would have the following Fourier decomposition:

$$
\gamma^{\prime 1}=\sum_{n \text { odd }}\left\{a_{n} \sigma_{0} \sin n \omega t+b_{n} \sigma_{0} \cos n \omega t\right\}
$$

Using equation C3 we can obtain a relation between the sine terms in Eq. C5 at $t_{1}$ and $t_{2}$ :

$$
\sin n \omega t_{1}=\sin \left(-n \omega t_{2}\right)=-\sin n \omega t_{2}
$$

Where the last equality follows from the odd property of the sine function. It is therefore

impossible for $\gamma^{\prime 1}\left(t_{1}\right)=\gamma^{\prime 1}\left(t_{2}\right)$ unless all the $b_{n}$ are zero. This leaves us only nonzero $a_{n}$. 
Note that any choice of $a_{n}$ will give a $\gamma^{\prime 1}$ which is a single-valued function of stress, however by the properties of the strain decomposition we can obtain the viscoplastic strain through the following:

$$
\gamma^{\prime \prime 1}=\gamma-\gamma^{1}
$$

The only way that $\dot{\gamma}^{\prime \prime 1}$ will also be a single-valued function of stress is if $a_{n}=J_{n}^{\prime}$ - i.e. we can only have sine terms in $\gamma^{\prime \prime 1}$ such that $\dot{\gamma}^{\prime \prime 1}$ will only contain cosine terms.

\section{REFERENCES}

Alexandrou, A. N., Constantinou, N., and Georgiou, G., "Shear rejuvenation, aging and shear banding in yield stress fluids," Journal of Non-Newtonian Fluid Mechanics 158, 6-17 (2009).

Ames, N. M., Srivastava, V., Chester, S., and Anand, L., "A thermo-mechanically coupled theory for large deformations of amorphous polymers. Part II: Applications," International Journal of Plasticity 25, 1495-1539 (2009).

Anand, L., Ames, N. M., Srivastava, V., and Chester, S., "A thermo-mechanically coupled theory for large deformations of amorphous polymers. Part I: Formulation," International Journal of Plasticity 25, 1474-1494 (2008).

Armstrong, P. J. and Frederick, C. O., "A mathematical representation of the multiaxial Bauschinger effect," Materials at High Temperatures 24, 1-26 (1966).

Atalik, K. and Keunings, R., "On the occurrence of even harmonics in the shear stress response of viscoelastic fluids in large amplitude oscillatory shear," Journal of NonNewtonian Fluid Mechanics 122, 107-116 (2004).

Ballesta, P., Besseling, R., Isa, L., Petekidis, G., and Poon, W. C. K., "Slip and Flow of Hard-Sphere Colloidal Glasses," Physical Review Letters 101 (2008).

Barnes, H. A., "A review of the slip (wall depletion) of polymer solutions, emulsions and particle suspensions in viscometers: its cause, character, and cure," Journal of Non-Newtonian Fluid Mechanics 56, 221-251 (1995).

Barnes, H. A., "Thixotropy - A review," Journal of Non-Newtonian Fluid Mechanics 70, 1-33 (1997).

Barnes, H. A., "The yield stress - a review or $\pi \alpha \nu \tau \alpha \rho \varepsilon \iota$ - everything flows?." Journal of Non-Newtonian Fluid Mechanics 81, 133 - 178 (1999).

Barral, Q., Ovarlez, G., Chateau, X., Boujlel, J., Rabideau, B., and Coussot, P., “Adhesion of yield stress fluids," Soft Matter 6, 1343-1351 (2010). 
Bauschinger, J., "Über die veränderung der position der elastizitätsgrenze des eisens und stahls durch strecken und quetschen und durch erwärmen und abkühlen und durch oftmals wiederholte beanspruchungen," Mitteilung aus dem Mechanisch-technischen Laboratorium der Königlichen polytechnischen Hoschschule in München 13, 1-115 (1886).

Bird, R. B., Armstrong, R. C., and Hassager, O., Dynamics of Polymeric Liquids Vol. 1, 2nd ed. (John Wiley and Sons, 1987).

Calin, A., Wilhelm, M., and Balan, C., "Determination of the non-linear parameter (mobility factor) of the Giesekus constitutive model using LAOS procedure," Journal of NonNewtonian Fluid Mechanics 165, 1564 - 1577 (2010).

Carnali, J. O. and Naser, M. S., "The use of dilute solution viscometry to characterize the network properties of Carbopol microgels," Colloid \& Polymer Science 270, 183-193 (1992).

Cho, Kwang Soo, Hyun, Kyu, Ahn, Kyung Hyun, and Lee, Seung Jong, "A geometrical interpretation of large amplitude oscillatory shear response," Journal of Rheology 49, 747-758 (2005).

Coussot, P., "Rheophysics of pastes: a review of microscopic modelling approaches," Soft Matter 3, 528-540 (2007).

Coussot, P., Nguyen, Q. D., Huynh, H. T., and Bonn, D., "Viscosity bifurcation in thixotropic, yielding fluids," Journal of Rheology 46, 573-589 (2002).

Coussot, P., Tocquer, L., Lanos, C., and Ovarlez, G., "Macroscopic vs. local rheology of yield stress fluids," Journal of Non-Newtonian Fluid Mechanics 158, 85-90 (2009).

Dimitriou, C. J., Casanellas, L., Ober, T. J., and McKinley, G. H., "Rheo-PIV of a shearbanding wormlike micellar solution under large amplitude oscillatory shear," Rheologica Acta 51, 395-411 (2012).

Dimitriou, C. J., McKinley, G. H., and Venkatesan, R., "Rheo-PIV analysis of the yielding and flow of model waxy crude oils," Energy \& Fuels 25, 3040-3052 (2011).

Divoux, T., Tamarii, D., Barentin, C., and Manneville, S., "Transient shear banding in a simple yield stress fluid," Physical Review Letters 104, 208301 (2010).

Doraiswamy, D., Mujumdar, A. N., Tsao, I., Beris, A. N., Danforth, S. C., and Metzner, A. B., "The Cox-Merz rule extended: A rheological model for concentrated suspensions and other materials with a yield stress," Journal of Rheology 35, 647-685 (1991).

Ewoldt, R. H., Nonlinear viscoelastic materials: Bioinspired applications and new characterization measures, Ph.D. thesis, Massachusetts Institute of Technology (2009).

Ewoldt, R. H., Clasen, C., Hosoi, A. E., and McKinley, G. H., "Rheological fingerprinting of gastropod pedal mucus and synthetic complex fluids for biomimicking adhesive locomotion," Soft Matter 3, 634-643 (2007). 
Ewoldt, R. H., Hosoi, A. E., and McKinley, G. H., "New measures for characterizing nonlinear viscoelasticity in large amplitude oscillatory shear," Journal of Rheology 52, 1427-1458 (2008).

Ewoldt, R. H., Winter, P., Maxey, J., and McKinley, G. H., "Large amplitude oscillatory shear of pseudoplastic and elastoviscoplastic materials," Rheologica Acta 49, 191-212 (2010).

Ferry, J. D., Viscoelastic Properties of Polymers, 3rd ed. (John Wiley and Sons, 1980).

Fielding, S. M., Cates, M. E., and Sollich, P., "Aging and rheology in soft materials," Journal of Rheology 44, 323-369 (2000).

Fielding, S. M., Cates, M. E., and Sollich, P., "Shear banding, aging and noise dynamics in soft glassy materials," Soft Matter 5, 2378-2382 (2009).

Ganeriwala, S. N. and Rotz, C. A., "Fourier transform mechanical analysis for determining the nonlinear viscoelastic properties of polymers," Polymer Engineering \& Science 27, 165-178 (1987).

Giacomin, A. J., Jeyaseelan, R. S., Samurkas, T., and Dealy, J. M., "Validity of separable BKZ model for large amplitude oscillatory shear," Journal of Rheology 37, 811-826 (1993).

Giacomin, A. J., Bird, R.B., Johnson, L.M., and Mix, A.W., "Large-amplitude oscillatory shear flow from the corotational Maxwell model," Journal of Non-Newtonian Fluid Mechanics 166, 1081 - 1099 (2011).

Gibaud, T., Barentin, C., Taberlet, N., and Manneville, S., "Shear-induced fragmentation of Laponite suspensions," Soft Matter 5, 3026-3037 (2009).

Graham, M. D., "Wall slip and the nonlinear dynamics of large-amplitude oscillatory shear flows," Journal of Rheology 39, 697-712 (1995).

Gurnon, A. K. and Wagner, N. J., "Large amplitude oscillatory shear (LAOS) measurements to obtain constitutive equation model parameters: Giesekus model of banding and nonbanding wormlike micelles," Journal of Rheology 56, 333-351 (2012).

Gurtin, M., Fried, E., and Anand, L., The Mechanics and Thermodynamics of Continua (Cambridge, 2010).

Henann, D. L. and Anand, L., "A large deformation theory for rate-dependent elasticplastic materials with combined isotropic and kinematic hardening," International Journal of Plasticity 25, 1833 - 1878 (2009).

Hess, A. and Aksel, N., "Yielding and structural relaxation in soft materials: Evaluation of strain-rate frequency superposition data by the stress decomposition method," Physical Review E 84, 051502 (2011).

Kalelkar, C., Lele, A., and Kamble, S., "Strain-rate frequency superposition in largeamplitude oscillatory shear," Physical Review E 81, 031401 (2010). 
Ketz, R. J., Prud'homme, R. K., and Graessley, W. W., "Rheology of concentrated microgel solutions," Rheologica Acta 27, 531-539 (1988).

Khan, A. S. and Huang, S., Continuum Theory of Plasticity (Wiley, 1995).

Klein, C. O., Spiess, H. W., Calin, A., Balan, C., and Wilhelm, M., "Separation of the nonlinear oscillatory response into a superposition of linear, strain hardening, strain softening, and wall slip response," Macromolecules 40, 4250-4259 (2007).

Kroner, E., "Allgemeine kontinuumstheorie der versetzungen und eigenspannungen," Archive for Rational Mechanics and Analysis 4, 273-334 (1960).

Läuger, J. and Stettin, H., "Differences between stress and strain control in the non-linear behavior of complex fluids," Rheologica Acta 49, 909-930 (2010).

Lemaitre, J. and Chaboche, J.-L., Mechanics of Solid Materials (Cambridge, 1990).

Macosko, C. W., Rheology: Principles, Measurements, and Applications (Wiley, 1994).

Mahaut, F., Chateau, X., Coussot, P., and Ovarlez, G., "Yield stress and elastic modulus of suspensions of noncolloidal particles in yield stress fluids," Journal of Rheology 52, 287-313 (2008).

Meeker, S. P., Bonnecaze, R. T., and Cloitre, M., "Slip and flow in pastes of soft particles: Direct observation and rheology," Journal of Rheology 48, 1295-1320 (2004a).

Meeker, S. P., Bonnecaze, R. T., and Cloitre, M., "Slip and flow in soft particle pastes," Physical Review Letters 92, 198302 (2004b).

Menut, P., Seiffert, S., Sprakel, J., and Weitz, D. A., "Does size matter? Elasticity of compressed suspensions of colloidal- and granular-scale microgels," Soft Matter 8, 156164 (2012).

Møller, P. C. F., Fall, A., and Bonn, D., "Origin of apparent viscosity in yield stress fluids below yielding," Europhysics Letters 87, 38004 (2009).

Møller, P. C. F., Fall, A., Chikkadi, V., Derks, D., and Bonn, D., "An attempt to categorize yield stress fluid behavior," Phil. Trans. R. Soc. A 367, 5139-5155 (2009).

Møller, P. C. F., Mewis, J, and Bonn, D, "Yield stress and thixotropy: On the difficulty of measuring yield stresses in practice," Soft Matter 2, 274-283 (2006).

Mujumdar, A., Beris, A. N., and Metzner, A. B., "Transient phenomena in thixotropic systems," Journal of Non-Newtonian Fluid Mechanics 102, 157-178 (2002).

Ng, T. S. K., McKinley, G. H., and Ewoldt, R. H., "Large amplitude oscillatory shear flow of gluten dough: A model power-law gel," Journal of Rheology 55, 627-654 (2011).

Oppong, F. K. and de Bruyn, J. R., "Diffusion of microscopic tracer particles in a yield-stress fluid," Journal of Non-Newtonian Fluid Mechanics 142, $104-111$ (2007).

Piau, J. M., "Carbopol gels: Elastoviscoplastic and slippery glasses made of individual swollen sponges meso- and macroscopic properties, constitutive equations and scaling laws," Journal of Non-Newtonian Fluid Mechanics 144, 1-29 (2007). 
Pipkin, A. C., Lectures on Viscoelasticity Theory (Springer, 1972).

Plazek, D. J., "What's wrong with the moduli Charley Brown? or Get the H out and go to L," Journal of Rheology 36, 1671-1690 (1992).

Roberts, G. P. and Barnes, H. A., "New measurements of the flow-curves for Carbopol dispersions without slip artefacts," Rheologica Acta 40, 499-503 (2001).

Rogers, S. A., Erwin, B. M., Vlassopoulos, D., and Cloitre, M., "Oscillatory yielding of a colloidal star glass," (2011a).

Rogers, S. A., Erwin, B. M., Vlassopoulos, D., and Cloitre, M., "A sequence of physical processes determined and quantified in LAOS: Application to a yield stress fluid," (2011b).

Rogers, S. A. and Lettinga, M. P., "A sequence of physical processes determined and quantified in large-amplitude oscillatory shear (LAOS): Application to theoretical nonlinear models," Journal of Rheology 56, 1-25 (2012).

Rubin, M. B. and Yarin, A. L., "On the relationship between phenomenological models for elastic-viscoplastic metals and polymeric liquids," Journal of Non-Newtonian Fluid Mechanics 50, 79 - 88 (1993).

Saramito, P., "A new elastoviscoplastic model based on the Herschel-Bulkley viscoplastic model," Journal of Non-Newtonian Fluid Mechanics 158, 154 - 161 (2009).

Sollich, P., "Rheological constitutive equation for a model of soft glassy materials," Physical Review E 58, 738-759 (1998).

de Souza Mendes, P. R., "Thixotropic elasto-viscoplastic model for structured fluids," Soft Matter 7, 2471-2483 (2011).

Wilhelm, M., "Fourier-transform rheology," Macromolecular Materials and Engineering 287, 83-105 (2002).

Wilhelm, M., Maring, D., and Spiess, H.-W., "Fourier-transform rheology," Rheologica Acta 37, 399-405 (1998).

Yoshimura, A. S. and Prud'homme, R. K., "Response of an elastic Bingham fluid to oscillatory shear," Rheologica Acta 26, 428-436 (1987).

Yziquel, F., Carreau, P. J., Moan, M., and Tanguy, P. A., "Rheological modeling of concentrated colloidal suspensions," Journal of Non-Newtonian Fluid Mechanics 86, 133 - 155 (1999).

Zhou, L., Cook, L. P., and McKinley, G. H., "Probing shear-banding transitions of the VCM model for entangled wormlike micellar solutions using large amplitude oscillatory shear (LAOS) deformations," Journal of Non-Newtonian Fluid Mechanics 165, 1462 $1472(2010)$. 